\title{
Adaptive Controller Design and Disturbance Attenuation for SISO Linear Systems with Zero Relative Degree under Noisy Output Measurements*
}

\author{
Sheng Zeng ${ }^{\dagger} \quad \mathrm{Yu}$ Chen ${ }^{\ddagger} \quad$ Zigang Pan ${ }^{\S}$
}

\begin{abstract}
In this paper, we present robust adaptive controller design for SISO linear systems with zero relative degree under noisy output measurements. We formulate the robust adaptive control problem as a nonlinear $H^{\infty}$-optimal control problem under imperfect state measurements, and then solve it using game theory. By using the a priori knowledge of the parameter vector, we apply a soft projection algorithm, which guarantees the robustness property of the closedloop system without any persistency of excitation assumption of the reference signal. Due to our formulation in state space, we allow the true system to be uncontrollable, as long as the uncontrollable part is stable in the sense of Lyapunov, and the uncontrollable modes on the $j \omega$-axis are uncontrollable from the exogenous disturbance input. This assumption allows the adaptive controller to asymptotically cancel out, at the output, the effect of exogenous sinusoidal disturbance inputs with unknown magnitude, phase, and frequency. These strong robustness properties are illustrated by a numerical example.
\end{abstract}

Keywords Nonlinear $H^{\infty}$ control; cost-to-come function analysis; robust adaptive control.

\section{Introduction}

The design of adaptive controllers has been an important research topic since 1970s. The classic adaptive control design, based on the certainty equivalence principle (Goodwin and Sin 1984, Goodwin and Mayne 1987), is to design the controller as if the system parameters are known and then in implementation to supply the controller with estimates of the parameters, using standard identifiers, as if the estimates are true values. This design method has been proven successful especially for the linear systems with or without stochastic disturbance inputs (Morse 1980, Kumar 1985, Narendra and Annaswamy 1989, Guo and Chen 1991, Ren and Kumar 1992, Naik et al. 1992, Guo 1996, Hsu et al. 1999). This approach leads to structurally simple adaptive controllers. Yet,

\footnotetext{
* Manuscript completed December 21, 2004. Research supported in part by the National Science Foundation under CAREER Award ECS-0296071.

${ }^{\dagger}$ Department of Electrical and Computer Engineering and Computer Science, University of Cincinnati, Cincinnati, OH 45221-0030. Tel: 513-556-1036; Email: zengs@ececs.uc.edu.

${ }^{\ddagger}$ Department of Electrical and Computer Engineering and Computer Science, University of Cincinnati, Cincinnati, OH 45221-0030. Tel: 513-556-1036; Email: cheny4@ececs.uc.edu.

${ }^{\S}$ Department of Electrical and Computer Engineering and Computer Science, 814 Rhodes Hall, Mail Loc. 0030, University of Cincinnati, Cincinnati, OH 45221-0030. Tel: 513-556-1808; Fax: 513-556-7326; Email: zpan@ececs.uc.edu. All correspondences should be addressed to this author.
} 
early designs based on this approach has been shown to be nonrobust (Ioannou and Kokotović 1983, Rohrs et al. 1985) when the system is subject to exogenous disturbance inputs and unmodeled dynamics. Then, the stability and the performance of a system under disturbance and/or uncertainty becomes an important issue. This motivates the study of robust adaptive control which has attracted significant research attention since 1980s. Also, this approach fails to generalize to systems with severe nonlinearities. This motivates the study of nonlinear adaptive control in 1990s.

Robust adaptive control has been an important research topic in late 1980s and early 1990s. Various adaptive controllers were modified to render the closed-loop systems robust (Datta and Ioannou 1994, Ioannou and Sun 1996). Despite their successes, they fell short of directly addressing the disturbance attenuation property of the closed-loop system.

The topic of nonlinear adaptive control has been widely studied in the last decade after the celebrated characterization of feedback linearizable or partially feedback linearizable systems (Isidori 1995). The introduction of the integrator backstepping methodology (Kanellakopoulos et al. 1991) allow us to design adaptive controllers for parametric strict-feedback and parametric pure-feedback nonlinear systems systematically. Since then, a lot of important contributions were motivated by this approach, and a complete list of references can be found in the book (Krstić et al. 1995). More recently, systems with unknown sign of the high frequency gain have been studied using this approach (Ye 2001). Moreover, this approach has been applied to linear systems to compare performance with the certainty equivalence approach (Krstić et al. 1994). As to be expected, a systematically designed nonlinear adaptive control law leads to better closed-loop performance than that for the certainty equivalence based design when the system is free of disturbance. However, this approach has also been shown to be nonrobust (Başar et al. 1996) when the system is subject to exogenous disturbance inputs.

$H^{\infty}$-optimal control has been proposed as a solution to the robust control problem. It achieves the objectives of robust control, namely, improving transient response, accommodating unmodeled dynamics, and rejecting exogenous disturbance inputs, by studying only the disturbance attenuation property for the closed-loop system. The game-theoretic approach to $H^{\infty}$-optimal control (Başar and Bernhard 1995) developed for the linear quadratic problems, offers the most promising tool to generalize the results to nonlinear systems (van der Schaft 1991, 1992, Isidori and Astolfi 1992, Didinsky et al. 1993, Didinsky 1994, Marino et al. 1994, Isidori and Kang 1995). The worstcase analysis approach to adaptive control was proposed to address the disturbance attenuation properties of the closed-loop system directly. In this approach, the robust adaptive control problem is formulated as a nonlinear $H^{\infty}$-optimal control problem under imperfect state measurements. Using cost-to-come function analysis, it can be converted into a problem under full information measurements. This full information measurement problem is then solved for a suboptimal solution using the integrator backstepping methodology. This design paradigm has been applied to worstcase parameter identification problems (Didinsky et al. 1995, Pan and Başar 1996), which has led to new classes of parametrized identifiers for linear and nonlinear systems. It has also been applied to adaptive control problems (Didinsky and Başar 1997, Pan and Başar 1998, Tezcan and Başar 1999, Pan and Başar 2000, Arslan and Başar 2001, Zeng and Pan 2003), which has led to new classes of parametrized robust adaptive controllers for linear and nonlinear systems. Adaptive control for strict-feedback systems was studied in Pan and Başar (1998) under noiseless full state measurements. More general class of nonlinear systems was studied in Arslan and Başar (2001), again with noiseless full state measurements. In Tezcan and Başar (1999), adaptive control for strict-feedback nonlinear systems was considered under noiseless output measurements. In Pan 
and Başar (2000) and Zeng and Pan (2003), linear system was considered under noisy output measurements. Zeng and Pan (2003) generalizes the results of Pan and Başar (2000), by assuming part of the disturbance inputs are measured, which then lead to disturbance feedforword structure in the adaptive controller.

In this paper, we study the adaptive control design for SISO linear systems with zero relative degree under noisy output measurements using a similar approach as that of Pan and Başar (2000). We assume that the linear system admits a known upper bound for its dynamic order, is observable, has a strictly minimum phase transfer function with relative degree 0 . The linear system may be uncontrollable, as long as the uncontrollable part is stable in the sense of Lyapunov, and all uncontrollable modes on the $j \omega$-axis are uncontrollable from the disturbance input. Under these assumptions, the system may be transformed into the design model, which is linear in all of the unknown quantities. We formulate the robust adaptive control problem as a nonlinear $H^{\infty}$-optimal control problem under imperfect state measurements, where the objectives of asymptotic tracking, transient performance, and disturbance attenuation are incorporated into a single game theoretic cost function. To avoid singularity is the estimation step, we assume that the measurement is noisy. Then, we can apply cost-to-come function methodology to derive the estimator, which has a finitedimensional structure. To relieve the persistency of excitation condition for the closed-loop system, we assume that the true value of the parameter vector belongs to a convex compact set characterized by a known smooth nonnegative radially unbounded and strictly convex function $P(\theta)$, and apply a soft projection algorithm for the estimator. Then, the closed-loop system is robust with or without the persistently exciting signals. After the estimator is determined, the original problem becomes a nonlinear $H^{\infty}$-optimal control problem under full-information measurement, and the controller can be obtained directly based on the cost function at that step. The closed-loop system admits a guaranteed disturbance attenuation level with respect to the exogenous disturbance inputs, where the ultimate attenuation lower bound for the achievable performance level is equal to the noise intensity in the measurement channel. All closed-loop signals are bounded for bounded disturbance input and bounded reference trajectory. Furthermore, it achieves asymptotic tracking of uniformly continuous and bounded reference trajectories for all bounded disturbance inputs that are of finite energy. This result has significant impact on active noise cancellation problems. That is, when the true system is subject to disturbances generated by an unknown exogenous linear system, we can extend our system model to include the states of the exogenous system as part of the model, and then asymptotically cancel out the effect of the noise at the output. This feature is illustrated by an example in the paper.

The balance of the paper is organized as follows. In Section 2, we list the notations to be used in this paper. In Section 3, we formulate adaptive control problem and discuss the general solution methodology. In Section 4, we present the estimation and control design using cost-tocome function methodology. In Section 5, we present the main result of the paper which states the robustness properties of the closed-loop system. The theoretical results are illustrated by a numerical example in Section 6. The paper ends with some concluding remarks in Section 7, and two appendices.

\section{Notations}

We denote $\mathbb{R}$ to be the real line; $\mathbb{N}$ to be the set of natural numbers; $\mathbb{C}$ to be the set of complex numbers. For a function $f$, we say that it belongs to $\mathcal{C}$ if it is continuous; we say that it belongs to 
$\mathcal{C}_{k}$ if it is $k$-times continuously (partial) differentiable. For any matrix $A, A^{\prime}$ denotes its transpose. For any $b \in \mathbb{R}, \operatorname{sgn}(b)=\left\{\begin{array}{cc}-1 & b<0 \\ 0 & b=0 \\ 1 & b>0\end{array}\right.$. For any vector $z \in \mathbb{R}^{n}$, where $n \in \mathbb{N},|z|$ denotes $\left(z^{\prime} z\right)^{1 / 2}$. For any vector $z \in \mathbb{R}^{n}$, and any $n \times n$-dimensional symmetric matrix $M$, where $n \in \mathbb{N}$, $|z|_{M}^{2}=z^{\prime} M z$. For any matrix $M$, the vector $\vec{M}$ is formed by stacking up its column vectors. For any symmetric matrix $M, \overleftarrow{M}$ denotes the vector formed by stacking up the column vector of the lower triangular part of $M$. For $n \times n$-dimensional symmetric matrices $M_{1}$ and $M_{2}$, where $n \in \mathbb{N}$, we write $M_{1}>M_{2}$ if $M_{1}-M_{2}$ is positive definite; we write $M_{1} \geq M_{2}$ if $M_{1}-M_{2}$ is positive semi-definite. For $n \in \mathbb{N}$, the set of $n \times n$-dimensional positive definite matrices is denoted by $\mathcal{S}_{+n}$. For $n \in \mathbb{N} \cup\{0\}, I_{n}$ denotes the $n \times n$-dimensional identity matrix. For any matrix $M,\|M\|_{p}$ denotes its $p$-induced norm, $1 \leq p \leq \infty$. $\mathcal{L}_{2}$ denotes the set of square integrable functions and $\mathcal{L}_{\infty}$ denotes the set of bounded functions. For any $n, m \in \mathbb{N}, \mathbf{0}_{n \times m}$ denotes the $n \times m$-dimensional matrix whose elements are zeros.

\section{Problem Formulation}

We consider the adaptive control problem for single-input and single-output (SISO) linear timeinvariant systems. We make the following assumption on the unknown system.

Assumption 1 The linear system is known to be at most $n$ dimensional, $n \in \mathbb{N}$.

We consider the following true system dynamics:

$$
\begin{aligned}
\grave{\dot{x}} & =\grave{A} \grave{x}+\grave{B} u+\grave{D} \grave{w} ; \quad \grave{x}(0)=\grave{x}_{0} \\
y & =\grave{C} \grave{x}+b_{0} u+\grave{E} \grave{w}
\end{aligned}
$$

where $\grave{x}$ is the $\grave{n}$-dimensional state vector, $\grave{n} \in \mathbb{N} \cup\{0\} ; u$ is the scalar control input; $b_{0} \in \mathbb{R}$ and $b_{0} \neq 0 ; y$ is the scalar measurement output; $\grave{w}$ is the $\grave{q}$-dimensional unmeasured disturbance input vector, $\grave{q} \in \mathbb{N}$; the state $\grave{x}$ has initial condition $\grave{x}_{0}$; and all input and output signals $y, u$, and $\grave{w}$ are continuous; the matrices $\grave{A}, \grave{B}, \grave{C}, \grave{D}$, and $\grave{E}$ are of the appropriate dimensions, generally unknown or partially unknown. The transfer function from $u$ to $y$ is $H(s)=\grave{C}\left(s I_{\grave{n}}-\grave{A}\right)^{-1} \grave{B}+b_{0}$.

Assumption $2^{1}$ The pair $(\grave{A}, \grave{C})$ is observable. The transfer function $H(s)$ is known to have relative degree 0 , and is strictly minimum phase. Moreover, the uncontrollable part (with respect to u) of the unknown system is stable in the sense of Lyapunov. Any uncontrollable mode corresponding to the eigenvalues of the matrix $\grave{A}$ on the $j \omega$-axis are uncontrollable from $\grave{w}$.

Remark 1 If the true system is of order $\grave{n}<n$, we can add $(n-\grave{n})$-dimensional dynamics as outlined in Lemma 6, such that the expanded system is of order $n$ and satisfies the Assumption 2. Hence, without loss of generality, we will assume that the true system (1) is of order $n$.

Since $(\grave{A}, \grave{C})$ is observable, there always exists a state diffeomorphism $x=\grave{T} \grave{x}$, and a disturbance transformation $w=\grave{M} \grave{w}$, where $\grave{T}$ is an unknown real invertible matrix and $\grave{M}$ is a real $q \times \grave{q}_{-}$ dimensional unknown matrix with $q \in \mathbb{N}$, such that the system (1) can be transformed into the

\footnotetext{
${ }^{1}$ When $\grave{n}=0$, Assumption 2 is considered satisfied.
} 
following form in the $x$ coordinate with inputs $u$ and $w$

$$
\begin{aligned}
\dot{x} & =A x+\left(y \bar{A}_{211}+u \bar{A}_{212}\right) \theta+B u+D w ; \quad x(0)=x_{0} \\
y & =C x+u \bar{C}_{1} \theta+b_{p 0} u+E w
\end{aligned}
$$

where $\theta$ is the $\sigma$-dimensional vector of unknown parameters of the system, $\sigma \in \mathbb{N}$; the matrices $A$, $\bar{A}_{211}, \bar{A}_{212}, B, D, C, E$, and $\bar{C}_{1}$ are of appropriate dimensions and completely known, and $b_{p 0} \in \mathbb{R}$ is also known. In addition, the high frequency gain of the transfer function $H(s), b_{0}$, is equal to $b_{p 0}+\bar{C}_{1} \theta$. We will design the adaptive controller based on system (2), which is called the design model. By Assumption 2, the pair $(A, C)$ is observable.

We have the following assumptions about the design model.

Assumption $3 E E^{\prime}>0$.

Define $\zeta:=\left(E E^{\prime}\right)^{-1 / 2}$ and $L:=D E^{\prime}$.

Remark 2 The above transformation matrix $\grave{T}$ always exists. One may choose the state diffeomorphism $x=\grave{T} \grave{x}$ to be the one which transforms the pair $(\grave{A}, \grave{C})$ into its observer canonical form. Next, we can choose a unknown matrix $\dot{M}$ such that the matrices $D$ and $E$ are completely known.

To guarantee the stability of the closed-loop system and the boundedness of the estimate of $\theta$, we make the following assumption on the parameter vector $\theta$.

Assumption 4 The sign of the high-frequency gain $b_{0}$ is known. There exists a known smooth nonnegative radially-unbounded strictly convex function $P: \mathbb{R}^{\sigma} \rightarrow \mathbb{R}$, such that the true value of $\theta$ belongs to the set $\Theta:=\left\{\bar{\theta} \in \mathbb{R}^{\sigma} \mid P(\bar{\theta}) \leq 1\right\}$. Furthermore, for any $\bar{\theta} \in \Theta$, we have $\operatorname{sgn}\left(b_{0}\right)\left(b_{p 0}+\right.$ $\left.\bar{C}_{1} \bar{\theta}\right)>0$.

We make the following assumption about the reference signal, $y_{d}$.

Assumption 5 The reference trajectory, $y_{d}$, is continuous, and available for the control design. $\diamond$

For the system (1), under Assumptions $1-5$, the control law is generated by

$$
u(t)=\mu\left(y_{[0, t]}, y_{d[0, t]}\right)
$$

Furthermore, it must satisfy the following condition. For any uncertainty $\left(x_{0}, \theta, \grave{w}_{[0, \infty)}, y_{d[0, \infty)}\right) \in$ $\grave{\mathcal{W}}:=\mathbb{R}^{n} \times \Theta \times \mathcal{C} \times \mathcal{C}$, which comprises the initial state, the true value of the unknown parameter vector, the unknown disturbance input waveform, and the reference trajectory, there must be a unique solution $\grave{x}_{[0, \infty)}$ for the closed-loop system, which results in a continuous control input waveform $u_{[0, \infty)}$. We denote the class of these admissible controllers by $\mathcal{M}_{u}$.

The objectives of our control design are to make the output of the system, $C x+b_{0} u$, to asymptotically track the reference trajectory $y_{d}$, and guarantee the boundedness of all closed-loop signals, while rejecting the uncertainty $\left(x_{0}, \theta, \grave{w}_{[0, \infty)}, y_{d[0, \infty)}\right) \in \mathcal{W}$. For design purposes, instead of attenuating the effect of $\grave{w}$, we design the adaptive controller to attenuate the effect of $w$. We take the uncertainty $\left(x_{0}, \theta, w_{[0, \infty)}, y_{d[0, \infty)}\right)$ to belong to the set $\mathcal{W}:=\mathbb{R}^{n} \times \Theta \times \mathcal{C} \times \mathcal{C}$. All of these objectives can be captured by the optimization of a single game-theoretic cost function, defined as follows. 
Definition 1 A controller $\mu \in \mathcal{M}_{u}$ is said to achieve disturbance attenuation level $\gamma$ if there exist a nonnegative function $l\left(t, \theta, x, y_{[0, t]}, y_{d[0, t]}\right)$ such that

$$
\sup _{\left(x_{0}, \theta, \grave{w}_{[0, \infty)}, y_{d[0, \infty)}\right) \in \dot{\mathcal{W}}} J_{\gamma t_{f}} \leq 0 ; \quad \forall t_{f} \geq 0
$$

where

$$
\begin{aligned}
J_{\gamma t_{f}}:= & \int_{0}^{t_{f}}\left(\left(C x(\tau)+u(\tau) \bar{C}_{1} \theta+b_{p 0} u(\tau)-y_{d}(\tau)\right)^{2}+l\left(\tau, \theta, x(\tau), y_{[0, \tau]}, y_{d[0, \tau]}\right)\right. \\
& \left.-\gamma^{2}|w(\tau)|^{2}\right) d \tau-\gamma^{2}\left|\left[\begin{array}{ll}
\theta^{\prime}-\check{\theta}_{0}^{\prime} & x_{0}^{\prime}-\check{x}_{0}^{\prime}
\end{array}\right]^{\prime}\right|_{\bar{Q}_{0}}^{2}
\end{aligned}
$$

where $\check{\theta}_{0} \in \Theta$ is the initial guess of the unknown parameter vector $\theta ; \check{x}_{0}$ is the initial guess of the unknown initial state $x_{0}$; the $(\sigma+n) \times(\sigma+n)$-dimensional matrix $\bar{Q}_{0}$ is the quadratic weighting on the initial estimation error, quantifying the level of confidence in the estimate $\left[\begin{array}{cc}\check{\theta}_{0}^{\prime} & \check{x}_{0}^{\prime}\end{array}\right]^{\prime} ; \bar{Q}_{0}^{-1}$ admits the structure $\left[\begin{array}{cc}Q_{0}^{-1} & Q_{0}^{-1} \Phi_{0}^{\prime} \\ \Phi_{0} Q_{0}^{-1} & \Pi_{0}+\Phi_{0} Q_{0}^{-1} \Phi_{0}^{\prime}\end{array}\right]$, where $Q_{0}$ and $\Pi_{0}$ are $\sigma \times \sigma$-and $n \times n$-dimensional positive definite matrices, respectively.

Clearly, when the inequality (4) is achieved, the squared $\mathcal{L}_{2}$ norm of the output tracking error $C x+u \bar{C}_{1} \theta+b_{p 0} u-y_{d}$ is bounded by $\gamma^{2}$ times the squared $\mathcal{L}_{2}$ norm of the transformed disturbance input $w$ plus some constant. When the $\mathcal{L}_{2}$ norm of $\grave{w}$ is finite, the squared $\mathcal{L}_{2}$ norm of $C x+u \bar{C}_{1} \theta+$ $b_{p 0} u-y_{d}$ is also finite, which implies $\lim _{t \rightarrow \infty}\left(C x(t)+u(t) \bar{C}_{1} \theta+b_{p 0} u(t)-y_{d}(t)\right)=0$, under additional assumptions.

The following notation will be used throughout this paper. Let $\check{x}$ denote the estimate of $x$, $\tilde{x}$ denote the state estimation error $x-\check{x}, \check{\theta}$ denote the estimate of $\theta, \tilde{\theta}$ denote the parameter estimation error $\theta-\check{\theta}$.

We intend to solve this robust adaptive control problem by formulating it as an $H^{\infty}$ control problem with imperfect state measurements. To do this, we first expand the state space to include the parameter $\theta$ as part of the state. Let $\xi$ denote the expanded state vector $\xi=\left[\begin{array}{ll}\theta^{\prime} & x^{\prime}\end{array}\right]^{\prime}$. Note that $\dot{\theta}=0$, we have the following expanded dynamics for system (2)

$$
\begin{aligned}
\dot{\xi} & =\left[\begin{array}{cc}
\mathbf{0}_{\sigma \times \sigma} & \mathbf{0}_{\sigma \times n} \\
y \bar{A}_{211}+u \bar{A}_{212} & A
\end{array}\right] \xi+\left[\begin{array}{c}
\mathbf{0}_{\sigma \times 1} \\
B
\end{array}\right] u+\left[\begin{array}{c}
\mathbf{0}_{\sigma \times q} \\
D
\end{array}\right] w \\
& =: \bar{A}(u, y) \xi+\bar{B} u+\bar{D} w \\
y & =\left[\begin{array}{ll}
u \bar{C}_{1} & C
\end{array}\right] \xi+b_{p 0} u+E w=: \bar{C}(u) \xi+b_{p 0} u+E w
\end{aligned}
$$

The worst-case optimization of the cost function (5) can be carried out in two steps as depicted in the following inequality.

$$
\begin{aligned}
& \sup _{\left(x_{0}, \theta, \grave{w}_{[0, \infty)}, y_{d[0, \infty)}\right) \in \mathcal{W}} J_{\gamma t_{f}}=\sup _{y_{[0, \infty)} \in \mathcal{C}, y_{d[0, \infty)} \in \mathcal{C}} \sup _{\left(x_{0}, \theta, \grave{w}_{[0, \infty)}, y_{d[0, \infty)}\right) \in \grave{\mathcal{W}} \mid y_{[0, \infty)}, y_{d[0, \infty)}} J_{\gamma t_{f}} \\
& \leq \sup _{y_{[0, \infty)} \in \mathcal{C}, y_{d[0, \infty)} \in \mathcal{C}} \sup _{\left(x_{0}, \theta, w_{[0, \infty)}, y_{d[0, \infty)}\right) \in \mathcal{W} \mid y_{[0, \infty)}, y_{d[0, \infty)}} J_{\gamma t_{f}}
\end{aligned}
$$


The inner supremum operator will be carried out first. It is the estimation design step, which will be presented in Section 4. Succinctly stated, in this step, we will calculate the maximum cost that is consistent with the given measurement waveform.

The outer supremum operator will be carried out second. It is the control design step, which will be discussed after the estimation design. In this step we design the control input $u$, which guarantees the robustness of the closed-loop system.

This completes the formulation of the robust adaptive control problem. Next, we turn to the estimation and control design in the next section.

\section{Estimation and Control Design}

In this section, we present the estimation and control design for the adaptive control problem formulated. The first step is estimation design. In this step, the measurement waveform $y_{[0, \infty)}$ and the reference trajectory $y_{d[0, \infty)}$ are assumed to be known. Since the control input is a causal function of $y$ and $y_{d}$, then it is also known. We apply the cost-to-come function methodology. Set function $l$ in (5) to $|\xi-\hat{\xi}|_{\bar{Q}}^{2}$, where $\hat{\xi}$ is the worst-case estimate for the expanded state $\xi, \hat{\xi}=\left[\begin{array}{ll}\theta^{\prime} & \hat{x}^{\prime}\end{array}\right]^{\prime}$, and $\bar{Q}$ is a matrix-valued weighting function to be introduced later. The cost function becomes

$$
\begin{aligned}
J_{\gamma t_{f}}= & \int_{0}^{t_{f}}\left(\left|C x(\tau)+u(\tau) \bar{C}_{1} \theta+b_{p 0} u(\tau)-y_{d}(\tau)\right|^{2}+|\xi(\tau)-\hat{\xi}(\tau)|_{\bar{Q}\left(\tau, y_{[0, \tau]}, y_{d[0, \tau]}\right)}^{2}\right. \\
& \left.-\gamma^{2}|w(\tau)|^{2}\right) d \tau-\gamma^{2}\left|\left[\begin{array}{cc}
\theta^{\prime}-\check{\theta}_{0}^{\prime} & x_{0}^{\prime}-\check{x}_{0}^{\prime}
\end{array}\right]^{\prime}\right|_{\bar{Q}_{0}}^{2}
\end{aligned}
$$

By the cost-to-come function analysis of Pan and Başar (2000), we have

$$
\begin{aligned}
\dot{\bar{\Sigma}}= & \left(\bar{A}(u, y)-\zeta^{2} \bar{L} \bar{C}(u)\right) \bar{\Sigma}+\bar{\Sigma}\left(\bar{A}(u, y)-\zeta^{2} \bar{L} \bar{C}(u)\right)^{\prime}+\gamma^{-2} \bar{D} \bar{D}^{\prime}-\gamma^{-2} \zeta^{2} \bar{L} \bar{L}^{\prime} \\
& -\bar{\Sigma}\left(\gamma^{2} \zeta^{2}(\bar{C}(u))^{\prime} \bar{C}(u)-(\bar{C}(u))^{\prime} \bar{C}(u)-\bar{Q}\left(t, y_{[0, t]}, y_{d[0, t]}\right)\right) \bar{\Sigma} ; \quad \bar{\Sigma}(0)=\gamma^{-2} \bar{Q}_{0}^{-1} \\
\dot{\check{\xi}}= & \bar{A}(u, y) \dot{\xi}-\bar{\Sigma} \bar{Q}\left(t, y_{[0, t]}, y_{d[0, t]}\right) \xi_{c}+\bar{\Sigma}(\bar{C}(u))^{\prime}\left(\bar{C}(u) \check{\xi}-\left(y_{d}-b_{p 0} u\right)\right)+\bar{B} u \\
& +\zeta^{2}\left(\gamma^{2} \bar{\Sigma}(\bar{C}(u))^{\prime}+\bar{L}\right)\left(y-b_{p 0} u-\bar{C}(u) \check{\xi}\right) ; \quad \check{\xi}(0)=\left[\begin{array}{ll}
\check{\theta}_{0}^{\prime} & \check{x}_{0}^{\prime}
\end{array}\right]^{\prime}
\end{aligned}
$$

where $\bar{L}$ is defined as $\bar{L}=\left[\begin{array}{ll}\mathbf{0}_{1 \times \sigma} & L^{\prime}\end{array}\right]^{\prime}$ and $\xi_{c}:=\hat{\xi}-\check{\xi}$.

Then, the cost function (8) can be equivalently written as, when $\bar{\Sigma}$ exists on $\left[0, t_{f}\right]$ and $\bar{\Sigma}(t)$ is positive definite $\forall t \in\left[0, t_{f}\right]$.

$$
\begin{aligned}
J_{\gamma t_{f}}= & -\left|\xi\left(t_{f}\right)-\check{\xi}\left(t_{f}\right)\right|_{\left(\bar{\Sigma}\left(t_{f}\right)\right)^{-1}}^{2}+\int_{0}^{t_{f}}\left(\left(\bar{C}(u(\tau)) \check{\xi}(\tau)+b_{p 0} u(\tau)-y_{d}(\tau)\right)^{2}\right. \\
& -\gamma^{2} \zeta^{2}\left(y(\tau)-b_{p 0} u(\tau)-\bar{C}(u(\tau)) \check{\xi}(\tau)\right)^{2}+|\hat{\xi}(\tau)-\check{\xi}(\tau)|_{\bar{Q}\left(\tau, y_{[0, \tau]}, y_{d[0, \tau]}\right)}^{2} \\
& \left.-\gamma^{2}\left|w(\tau)-w_{*}(\xi(\tau), \check{\xi}(\tau), \bar{\Sigma}(\tau), u(\tau), w(\tau))\right|^{2}\right) d \tau
\end{aligned}
$$

where $w_{*}: \mathbb{R}^{n+\sigma} \times \mathbb{R}^{n+\sigma} \times \mathcal{S}_{+(n+\sigma)} \times \mathbb{R} \times \mathbb{R}^{q} \rightarrow \mathbb{R}^{q}$ is the worst-case disturbance for estimation step, given by

$$
w_{*}(\xi, \check{\xi}, \bar{\Sigma}, u, w)=\zeta^{2} E^{\prime}\left(y-b_{p 0} u-\bar{C}(u) \xi\right)+\gamma^{-2}\left(I_{q}-\zeta^{2} E^{\prime} E\right) \bar{D}^{\prime} \bar{\Sigma}^{-1}(\xi-\check{\xi})
$$


The following steps of derivation for the estimator closely resembles that in Pan and Başar (2000). Partition $\bar{\Sigma}(t)$ as

$$
\bar{\Sigma}(t)=\left[\begin{array}{cc}
\Sigma(t) & \bar{\Sigma}_{12}(t) \\
\bar{\Sigma}_{21}(t) & \bar{\Sigma}_{22}(t)
\end{array}\right]
$$

where $\Sigma(t)$ is $\sigma \times \sigma$-dimensional, and introduce $\Phi(t):=\bar{\Sigma}_{21}(t)(\Sigma(t))^{-1}$ and $\Pi(t):=\gamma^{2}\left(\bar{\Sigma}_{22}(t)-\right.$ $\left.\bar{\Sigma}_{21}(t)(\Sigma(t))^{-1} \bar{\Sigma}_{12}(t)\right)$. Also partition $\check{\xi}$ compatibly as $\left[\begin{array}{cc}\check{\theta}^{\prime} & \check{x}^{\prime}\end{array}\right]$.

For the boundedness of $\Sigma$, the weighting matrix $\bar{Q}$ in (8) admits the following structure

$$
\begin{aligned}
\bar{Q}\left(t, y_{[0, t]}, y_{d[0, t]}\right)= & (\bar{\Sigma}(t))^{-1}\left[\begin{array}{cc}
\mathbf{0}_{\sigma \times \sigma} & \mathbf{0}_{\sigma \times n} \\
\mathbf{0}_{n \times \sigma} & \Delta(t)
\end{array}\right](\bar{\Sigma}(t))^{-1} \\
& +\left[\begin{array}{cc}
\epsilon(t)\left(\bar{C}_{1} u(t)+C \Phi(t)\right)^{\prime}\left(\gamma^{2} \zeta^{2}-1\right)\left(\bar{C}_{1} u(t)+C \Phi(t)\right) & \mathbf{0}_{\sigma \times n} \\
\mathbf{0}_{n \times \sigma} & \mathbf{0}_{n \times n}
\end{array}\right] \\
= & {\left[\begin{array}{c}
-(\Phi(t))^{\prime} \\
I_{n}
\end{array}\right] \gamma^{4}(\Pi(t))^{-1} \Delta(t)(\Pi(t))^{-1}\left[\begin{array}{c}
-(\Phi(t))^{\prime} \\
I_{n}
\end{array}\right] } \\
& +\left[\begin{array}{c}
\epsilon(t)\left(\bar{C}_{1} u(t)+C \Phi(t)\right)^{\prime}\left(\gamma^{2} \zeta^{2}-1\right)\left(\bar{C}_{1} u(t)+C \Phi(t)\right) \\
\mathbf{0}_{n \times \sigma}
\end{array}\right.
\end{aligned}
$$

where $\Delta(t)=\gamma^{-2} \beta_{\Delta} \Pi(t)+\Delta_{1}$, with $\beta_{\Delta} \geq 0$ being a constant and $\Delta_{1}$ being an $n \times n$ positive-definite matrix, and $\epsilon$ is a scalar function defined by

$$
\begin{aligned}
\epsilon(t) & :=K_{c}^{-1} s_{\Sigma}(t):=\operatorname{Tr}\left((\Sigma(t))^{-1}\right) / K_{c} \quad t \in[0, \infty) \\
\text { or } \epsilon(t) & :=1
\end{aligned}
$$

and $K_{c} \geq \gamma^{2} \operatorname{Tr}\left(Q_{0}\right)$ is a constant. Because of this structure for $\bar{Q}$, we will later treat $\bar{Q}$ as a function $\bar{Q}: \mathbb{R}^{n \times \sigma} \times \mathbb{R} \times \mathbb{R} \rightarrow \mathbb{R}^{(n+\sigma) \times(n+\sigma)}, \bar{Q}\left(\Phi, u, s_{\Sigma}\right)^{2}$.

Then, we have the following differential equation for $\Sigma, \Phi$, and $\Pi$

$$
\begin{aligned}
\dot{\Sigma}= & -(1-\epsilon) \Sigma\left(\bar{C}_{1} u+C \Phi\right)^{\prime}\left(\gamma^{2} \zeta^{2}-1\right)\left(\bar{C}_{1} u+C \Phi\right) \Sigma ; \quad \Sigma(0)=\gamma^{-2} Q_{0}^{-1} \\
\dot{\Phi}= & \left(A-\zeta^{2} L C-\Pi C^{\prime}\left(\zeta^{2}-\gamma^{-2}\right) C\right) \Phi+y \bar{A}_{211}+u\left(\bar{A}_{212}-\zeta^{2} L \bar{C}_{1}-\Pi C^{\prime}\left(\zeta^{2}-\gamma^{-2}\right) \bar{C}_{1}\right) ; \\
& \quad \Phi(0)=\Phi_{0} \\
\dot{\Pi}= & \left(A-\zeta^{2} L C+\beta_{\Delta} / 2 I_{n}\right) \Pi+\Pi\left(A-\zeta^{2} L C+\beta_{\Delta} / 2 I_{n}\right)^{\prime}-\Pi C^{\prime}\left(\zeta^{2}-\gamma^{-2}\right) C \Pi+D D^{\prime} \\
& \quad-\zeta^{2} L L^{\prime}+\gamma^{2} \Delta_{1} ; \quad \Pi(0)=\Pi_{0}
\end{aligned}
$$

The matrix $\Sigma$ will play the role of worst-case covariance matrix of the parameter estimation error. The choice of $\bar{Q}$ guarantees that $\Sigma$ is bounded from above and bounded from below away from 0 as depicted in the following Lemma, whose proof is given in Pan and Başar (2000).

Lemma 1 Consider the dynamic equation (15a) for the covariance matrix $\Sigma$. Let $K_{c} \geq \gamma^{2} \operatorname{Tr}\left(Q_{0}\right)$, $Q_{0}>0$, and $\gamma \geq \zeta^{-1}$. Then, the matrix $\Sigma$ is upper and lower bounded as follows; for either choice of $\epsilon(t)$ as in (14), whenever $\Sigma$ is defined on $\left[0, t_{f}\right]$, and $\Phi$ and $u$ are continuous on $\left[0, t_{f}\right]$.

$$
K_{c}^{-1} I_{\sigma} \leq \Sigma(t) \leq \Sigma(0)=\gamma^{-2} Q_{0}^{-1} ; \quad \gamma^{2} \operatorname{Tr}\left(Q_{0}\right) \leq \operatorname{Tr}\left((\Sigma(t))^{-1}\right) \leq K_{c} ; \quad \forall t \in\left[0, t_{f}\right]
$$

\footnotetext{
${ }^{2} \Pi(t)$ will be set as a constant by Assumption 7 .
} 
To avoid the inversion of $\Sigma$ on-line, we define $s_{\Sigma}(t):=\operatorname{Tr}\left((\Sigma(t))^{-1}\right)$, and its time derivative is given by

$$
\dot{s}_{\Sigma}=\left(\gamma^{2} \zeta^{2}-1\right)(1-\epsilon)\left(\bar{C}_{1} u+C \Phi\right)\left(\bar{C}_{1} u+C \Phi\right)^{\prime} ; \quad s_{\Sigma}(0)=\gamma^{2} \operatorname{Tr}\left(Q_{0}\right)
$$

Then, $\epsilon(t)=K_{c}^{-1} s_{\Sigma}(t)$, which does not require the inversion of $\Sigma(t)$, when $\epsilon$ is defined by (14a).

Based on Lemma 1, we note that $\gamma \geq \zeta^{-1}$. This means that the quantity $\zeta^{-1}$ is the ultimate lower bound on the achievable performance level for the adaptive system, using the design method proposed in this paper.

Assumption 6 If the matrix $A-\zeta^{2} L C$ is Hurwitz, then the desired disturbance attenuation level $\gamma \geq \zeta^{-1}$. In case $\gamma=\zeta^{-1}$, choose $\beta_{\Delta} \geq 0$ such that $A-\zeta^{2} L C+\beta_{\Delta} / 2 I_{n}$ is Hurwitz. If the matrix $A-\zeta^{2} L C$ is not Hurwitz, then the desired disturbance attenuation level $\gamma>\zeta^{-1}$.

Assumption 7 The initial weighting matrix $\Pi_{0}$ in (15c) is chosen as the unique positive definite solution to the algebraic Riccati equation:

$$
\left(A-\zeta^{2} L C+\beta_{\Delta} / 2 I_{n}\right) \Pi+\Pi\left(A-\zeta^{2} L C+\beta_{\Delta} / 2 I_{n}\right)^{\prime}-\Pi C^{\prime}\left(\zeta^{2}-\gamma^{-2}\right) C \Pi+D D^{\prime}-\zeta^{2} L L^{\prime}+\gamma^{2} \Delta_{1}=\mathbf{0}
$$

Then, we note that the unique positive-definite solution of $(15 \mathrm{c})$ is time-invariant and equal to the initial value $\Pi_{0}$, and the matrix $A_{f}:=A-\zeta^{2} L C-\Pi C^{\prime}\left(\zeta^{2}-\gamma^{-2}\right) C$ is Hurwitz.

To guarantee the boundedness of estimated parameters without persistently exciting signals, we introduce soft projection design on the parameter estimate, which is based on the a priori information on the bounds of the true value of the parameter vector $\theta$, i.e., Assumption 4.

Define

$$
\rho:=\inf \left\{P(\bar{\theta}) \mid \bar{\theta} \in \mathbb{R}^{\sigma} \text { and } b_{p 0}+\bar{C}_{1} \bar{\theta}=0\right\}
$$

By Assumption 4, we have $1<\rho \leq \infty$. Fix any $\rho_{o} \in(1, \rho)$, and define the open set $\Theta_{o}:=\{\bar{\theta} \in$ $\left.\mathbb{R}^{\sigma} \mid P(\bar{\theta})<\rho_{o}\right\}$. Our control design will guarantee the estimate $\check{\theta}$ lies in $\Theta_{o}$, which immediately implies $\check{b}_{0}:=b_{p 0}+\bar{C}_{1} \check{\theta} \geq c_{0}>0$ (See Zeng and Pan 2003). Moreover, the convexity of $P$ implies the following inequality

$$
\frac{\partial P}{\partial \theta}(\check{\theta})(\theta-\check{\theta})<0 \quad \forall \check{\theta} \in \mathbb{R}^{\sigma} \backslash \Theta
$$

Add the term $-\bar{\Sigma}\left[\left(P_{r}(\check{\theta})\right)^{\prime} \mathbf{0}_{1 \times n}\right]^{\prime}$ to the right-hand-side of the dynamics $(9 \mathrm{~b})$, where

$$
\begin{aligned}
& P_{r}(\check{\theta}):=\left\{\begin{array}{cl}
\frac{\exp \left(\frac{1}{1-P(\tilde{\theta})}\right)}{\left(\rho_{o}-P(\check{\theta})\right)^{3}}\left(\frac{\partial P}{\partial \theta}(\check{\theta})\right)^{\prime} & \forall \check{\theta} \in \Theta_{o} \backslash \Theta \\
\mathbf{0}_{\sigma \times 1} & \forall \check{\theta} \in \Theta
\end{array}\right. \\
& =: p_{r}(\check{\theta})\left(\frac{\partial P}{\partial \theta}(\check{\theta})\right)^{\prime}
\end{aligned}
$$


and $P_{r}(\check{\theta})$ and $p_{r}(\check{\theta})$ are smooth functions on $\Theta_{o}$. Then, we have

$$
\begin{aligned}
& \dot{\check{\xi}}=-\bar{\Sigma}\left[\begin{array}{ll}
\left(P_{r}(\check{\theta})\right)^{\prime} & \mathbf{0}_{1 \times n}
\end{array}\right]^{\prime}+\bar{A}(u, y) \check{\xi}-\bar{\Sigma} \bar{Q} \xi_{c}+\bar{\Sigma}(\bar{C}(u))^{\prime}(\bar{C}(u) \check{\xi} \\
& \left.-\left(y_{d}-b_{p 0} u\right)\right)+\bar{B} u+\zeta^{2}\left(\gamma^{2} \bar{\Sigma}(\bar{C}(u))^{\prime}+\bar{L}\right)\left(y-b_{p 0} u-\bar{C}(u) \check{\xi}\right) ; \\
& \check{\xi}(0)=\left[\begin{array}{ll}
\check{\theta}_{0}^{\prime} & \check{x}_{0}^{\prime}
\end{array}\right]^{\prime}
\end{aligned}
$$

Partition $\check{\xi}$ into $\left[\check{\theta}^{\prime} \check{x}^{\prime}\right]^{\prime}$ to obtain the dynamics for these individual estimates. We summarize the equations below.

$$
\begin{aligned}
& \left(A-\zeta^{2} L C+\beta_{\Delta} / 2 I_{n}\right) \Pi+\Pi\left(A-\zeta^{2} L C+\beta_{\Delta} / 2 I_{n}\right)^{\prime}-\Pi C^{\prime}\left(\zeta^{2}-\gamma^{-2}\right) C \Pi+D D^{\prime}-\zeta^{2} L L^{\prime}+\gamma^{2} \Delta_{1}=\mathbf{0} \\
& \dot{\Sigma}=-(1-\epsilon) \Sigma\left(\bar{C}_{1} u+C \Phi\right)^{\prime}\left(\gamma^{2} \zeta^{2}-1\right)\left(\bar{C}_{1} u+C \Phi\right) \Sigma ; \quad \Sigma(0)=\gamma^{-2} Q_{0}^{-1} \\
& \dot{s}_{\Sigma}=\left(\gamma^{2} \zeta^{2}-1\right)(1-\epsilon)\left(\bar{C}_{1} u+C \Phi\right)\left(\bar{C}_{1} u+C \Phi\right)^{\prime} ; \quad s_{\Sigma}(0)=\gamma^{2} \operatorname{Tr}\left(Q_{0}\right) \\
& A_{f}=A-\zeta^{2} L C-\Pi C^{\prime} C\left(\zeta^{2}-\gamma^{-2}\right) \\
& \dot{\Phi}=A_{f} \Phi+y \bar{A}_{211}+u\left(\bar{A}_{212}-\zeta^{2} L \bar{C}_{1}-\Pi C^{\prime}\left(\zeta^{2}-\gamma^{-2}\right) \bar{C}_{1}\right) ; \quad \Phi(0)=\Phi_{0} \\
& \dot{\tilde{\theta}}=-\Sigma P_{r}(\check{\theta})-\left[\begin{array}{ll}
\Sigma & \Sigma \Phi^{\prime}
\end{array}\right] \bar{Q} \xi_{c}-\left(\Sigma \bar{C}_{1}^{\prime} u+\Sigma \Phi^{\prime} C^{\prime}\right)\left(y_{d}-b_{p 0} u-\bar{C}_{1} \check{\theta} u-C \check{x}\right) \\
& +\gamma^{2} \zeta^{2}\left(\Sigma \bar{C}_{1}^{\prime} u+\Sigma \Phi^{\prime} C^{\prime}\right)\left(y-b_{p 0} u-\bar{C}_{1} \check{\theta} u-C \check{x}\right) ; \check{\theta}(0)=\check{\theta}_{0} \\
& \dot{\check{x}}=-\Phi \Sigma P_{r}(\check{\theta})+A \check{x}+\left(y \bar{A}_{211}+u \bar{A}_{212}\right) \check{\theta}+B u-\left[\Phi \Sigma \gamma^{-2} \Pi+\Phi \Sigma \Phi^{\prime}\right] \bar{Q} \xi_{c} \\
& +\zeta^{2}\left(\gamma^{2}\left(\Phi \Sigma \bar{C}_{1}^{\prime} u+\gamma^{-2} \Pi C^{\prime}+\Phi \Sigma \Phi^{\prime} C^{\prime}\right)+L\right)\left(y-b_{p 0} u-\bar{C}_{1} \check{\theta} u-C \check{x}\right) \\
& -\left(\Phi \Sigma \bar{C}_{1}^{\prime} u+\gamma^{-2} \Pi C^{\prime}+\Phi \Sigma \Phi^{\prime} C^{\prime}\right)\left(y_{d}-b_{p 0} u-\bar{C}_{1} \check{\theta} u-C \check{x}\right) ; \check{x}(0)=\check{x}_{0}
\end{aligned}
$$

To simplify the controller structure, the dynamics for $\Phi$ can be implemented with $3 n$ integrators instead of the $\sigma n$ integrators. First, we observe that the pair $\left(A_{f}, C\right)$ is observable. Then we introduce the matrix

$$
M_{f}:=\left[\begin{array}{llll}
A_{f}^{n-1} p_{n} & \cdots & A_{f} p_{n} & p_{n}
\end{array}\right]
$$

where $p_{n}$ is an $n$-dimensional vector such that the pair $\left(A_{f}, p_{n}\right)$ is controllable ${ }^{3}$, which implies that $M_{f}$ is invertible. Then the following $3 n$-dimensional prefiltering system for $y$ and $u$ generates the $\Phi$ online:

$$
\begin{aligned}
& \dot{\eta}=A_{f} \eta+p_{n} y ; \quad \eta(0)=\eta_{0} \\
& \dot{\lambda}=A_{f} \lambda+p_{n} u ; \quad \lambda(0)=\lambda_{0} \\
& \dot{\lambda}_{o}=A_{f} \lambda_{o} ; \quad \lambda_{o}(0)=p_{n} \\
& \Phi=\left[\begin{array}{llll}
A_{f}^{n-1} \eta & \cdots & A_{f} \eta & \eta
\end{array}\right] M_{f}^{-1} \bar{A}_{211}+\left[\begin{array}{llll}
A_{f}^{n-1} \lambda & \cdots & A_{f} \lambda & \lambda
\end{array}\right] M_{f}^{-1}\left(\bar{A}_{212}\right. \\
& \left.-\zeta^{2} L \bar{C}_{1}-\Pi C^{\prime}\left(\zeta^{2}-\gamma^{-2}\right) \bar{C}_{1}\right)+\left[\begin{array}{llll}
A_{f}^{n-1} \lambda_{o} & \cdots & A_{f} \lambda_{o} & \lambda_{o}
\end{array}\right] M_{f}^{-1} \Phi_{o 0}
\end{aligned}
$$

where $\eta_{0} \in \mathbb{R}^{n}, \lambda_{0} \in \mathbb{R}^{n}$, and $\Phi_{o 0} \in \mathbb{R}^{n \times \sigma}$ are such that (23d) holds at $t=0$.

Associated with the above identifier, introduce the value function, $W: \mathbb{R}^{n+\sigma} \times \mathbb{R}^{n+\sigma} \times$ $\mathcal{S}_{+(n+\sigma)} \rightarrow \mathbb{R}$,

$$
\begin{aligned}
W(\xi, \check{\xi}, \bar{\Sigma}) & =|\xi-\check{\xi}|_{\Sigma^{-1}}^{2} \\
& =|\theta-\check{\theta}|_{\Sigma^{-1}}^{2}+\gamma^{2}|x-\check{x}-\Phi(\theta-\check{\theta})|_{\Pi^{-1}}^{2}
\end{aligned}
$$

\footnotetext{
${ }^{3}$ The existence of $p_{n}$ is proven in Zhao and Pan (2003).
} 
whose time derivative along the dynamics of $\xi, \check{\xi}$, and $\bar{\Sigma}$ are given by

$$
\begin{aligned}
\dot{W}\left(\xi, \check{\xi}, \bar{\Sigma}, s_{\Sigma}, y_{d}, \hat{\xi}, u, w\right)= & -\left|C x+\bar{C}_{1} \theta u-\left(y_{d}-b_{p 0} u\right)\right|^{2}+\left|C \check{x}+\bar{C}_{1} \check{\theta} u-\left(y_{d}-b_{p 0} u\right)\right|^{2} \\
& -|\xi-\hat{\xi}|_{\bar{Q}}^{2}+\left|\check{\xi}-\hat{\xi}^{2}\right|_{\bar{Q}}^{2}+\gamma^{2}|w|^{2}-\gamma^{2}\left|w-w_{*}\right|^{2} \\
& -\gamma^{2} \zeta^{2}\left|y-b_{p 0} u-C \check{x}-\bar{C}_{1} \check{\theta} u\right|^{2}+2(\theta-\check{\theta})^{\prime} P_{r}(\check{\theta})
\end{aligned}
$$

which holds as long as $\Sigma>0, \check{\theta} \in \Theta_{o}$. We note that the last term in $\dot{W}$ is nonpositive, zero on the set $\Theta$ and approaches $-\infty$ as $\check{\theta}$ approaches the boundary of the set $\Theta_{o}$, which guarantees the boundness of $\check{\theta}$.

Then the cost function (8) can be equivalently written as, assuming $\Sigma(t)>0$ and $\check{\theta}(t) \in \Theta_{o}$, $\forall t \in\left[0, t_{f}\right]$,

$$
\begin{aligned}
J_{\gamma t_{f}}= & J_{\gamma t_{f}}+W(\xi(0), \check{\xi}(0), \bar{\Sigma}(0))-W\left(\xi\left(t_{f}\right), \check{\xi}\left(t_{f}\right), \bar{\Sigma}\left(t_{f}\right)\right)+\int_{0}^{t_{f}} \dot{W} d \tau \\
= & -\left|\xi\left(t_{f}\right)-\check{\xi}\left(t_{f}\right)\right|_{\left(\bar{\Sigma}\left(t_{f}\right)\right)^{-1}}^{2}+\int_{0}^{t_{f}}\left(\left(C \check{x}(\tau)+b_{p 0} u(\tau)+\bar{C}_{1} \check{\theta}(\tau) u(\tau)-y_{d}(\tau)\right)^{2}\right. \\
& -\gamma^{2} \zeta^{2}\left(y(\tau)-C \check{x}(\tau)-b_{p 0} u(\tau)-\bar{C}_{1} \check{\theta}(\tau) u(\tau)\right)^{2}+\left|\xi_{c}(\tau)\right|_{\bar{Q}\left(\Phi(\tau), u(\tau), s_{\Sigma}(\tau)\right)}^{2} \\
& \left.-\gamma^{2}\left|w(\tau)-w_{*}(\xi(\tau), \check{\xi}(\tau), \bar{\Sigma}(\tau), u(\tau), w(\tau))\right|^{2}+2(\theta-\check{\theta}(\tau))^{\prime} P_{r}(\check{\theta}(\tau))\right) d \tau
\end{aligned}
$$

This completes the estimation design step. Next, we consider the control design step.

Based on the inequality (7) in Section 3, the controller design is to guarantee that the following supremum is less than or equal to zero for all measurement waveforms,

$$
\begin{aligned}
& \sup _{\left(x_{0}, \theta, \grave{w}_{[0, \infty)}, y_{d[0, \infty)}\right) \in \mathcal{W}} J_{\gamma t_{f}} \\
& \leq \sup _{y_{[0, \infty)} \in \mathcal{C}, y_{d[0, \infty)} \in \mathcal{C}} J_{\left(x_{0}, \theta, w_{[0, \infty)}, y_{d[0, \infty)}\right) \in \mathcal{W} \mid y_{[0, \infty)}, y_{d[0, \infty)}} \sup _{\gamma t_{f}} \\
& \leq \sup _{y_{[0, \infty)} \in \mathcal{C}, y_{d[0, \infty)} \in \mathcal{C}}\left\{\int _ { 0 } ^ { t _ { f } } \left(\left(C \check{x}(\tau)+\left(b_{p 0}+\bar{C}_{1} \check{\theta}(\tau)\right) u(\tau)-y_{d}(\tau)\right)^{2}\right.\right. \\
& \left.\left.+\left|\xi_{c}(\tau)\right|_{\bar{Q}\left(\Phi(\tau), u(\tau), s_{\Sigma}(\tau)\right)}^{2}-\gamma^{2} \zeta^{2}\left(y(\tau)-C \check{x}(\tau)-\left(b_{p 0}+\bar{C}_{1} \check{\theta}(\tau)\right) u(\tau)\right)^{2}\right) d \tau\right\}
\end{aligned}
$$

By inequality (27), we observe that the cost function is expressed in terms of signals that we can measure or construct. This is then a nonlinear $H^{\infty}$-optimal control problem under full information measurements. Instead of considering $y$ as the maximizing variable, we can equivalently deal with the transformed variable:

$$
v:=\zeta\left(y-C \check{x}-\left(b_{p 0}+\bar{C}_{1} \check{\theta}\right) u\right)
$$

A clear choice for control input $u$, and the worst-case estimate $\hat{\xi}$, which gurantees that the right-hand-side of (27) is nonpositive, is

$$
\begin{aligned}
& u:=\bar{\mu}\left(\check{\theta}, \check{x}, y_{d}\right)=\frac{y_{d}-C \check{x}}{\bar{C}_{1} \check{\theta}+b_{p 0}} \\
& \hat{\xi}=\check{\xi}
\end{aligned}
$$


where $\bar{\mu}: \Theta_{o} \times \mathbb{R}^{n} \times \mathbb{R} \rightarrow \mathbb{R}$ is smooth. The value function for the closed-loop system is simply $W$, whose time derivative along solutions of the dynamics for $\xi, \check{\xi}$, and $\bar{\Sigma}$ is

$$
\begin{aligned}
\dot{W}= & -\left(C x+\bar{C}_{1} \theta u+b_{p 0} u-y_{d}\right)^{2}-|\xi-\hat{\xi}|_{\bar{Q}}^{2}+\gamma^{2}|w|^{2}-\gamma^{2}|v|^{2}-\gamma^{2}\left|w-w_{*}\right|^{2}+2(\theta-\check{\theta})^{\prime} P_{r}(\check{\theta}) \\
= & -\left(C x+\bar{C}_{1} \theta u+b_{p 0} u-y_{d}\right)^{2}-|\xi-\hat{\xi}|_{\bar{Q}}^{2}+\gamma^{2}|w|^{2}-\gamma^{2}\left|w-w_{o p t}\left(\xi, \check{\xi}, y_{d}, \bar{\Sigma}\right)\right|^{2} \\
& +2(\theta-\check{\theta})^{\prime} P_{r}(\check{\theta})
\end{aligned}
$$

where the worst-case disturbance with respect to the value function $W$ is given by, ${ }^{4} w_{\text {opt }}: \mathbb{R}^{n+\sigma} \times$ $\mathbb{R}^{n+\sigma} \times \mathbb{R} \times \mathcal{S}_{+(n+\sigma)} \rightarrow \mathbb{R}^{q}$

$$
w_{o p t}\left(\xi, \check{\xi}, y_{d}, \bar{\Sigma}\right)=-\zeta^{2} E^{\prime}\left[\bar{\mu}\left(\check{\theta}, \check{x}, y_{d}\right) \bar{C}_{1} \quad C\right](\xi-\check{\xi})+\gamma^{-2}\left(I_{q}-\zeta^{2} E^{\prime} E\right) \bar{D}^{\prime} \bar{\Sigma}^{-1}(\xi-\check{\xi})
$$

which holds as long as $\Sigma>0$ and $\check{\theta} \in \Theta_{o}$. Clearly, the closed-loop system is dissipative with storage function $W$ and supply rate

$$
-\left(C x+\bar{C}_{1} \theta u+b_{p 0} u-y_{d}\right)^{2}+\gamma^{2}|w|^{2}
$$

This completes the control design step. We will turn to present the main results in the next section.

\section{Main Result}

With the estimation and control design of the previous section, the state of the closed-loop system is given by

$$
X:=\left[\begin{array}{lllllll}
\theta^{\prime} & x^{\prime} & \overleftarrow{\Sigma}^{\prime} & s_{\Sigma} & \check{\theta}^{\prime} & \check{x}^{\prime} & \vec{\Phi}^{\prime}
\end{array}\right]^{\prime}
$$

which belongs to the open set

$$
\mathcal{D}:=\left\{X \mid \Sigma>0, s_{\Sigma}>0, \check{\theta} \in \Theta_{o}\right\}
$$

The dynamics for $X$ are

$$
\dot{X}=F\left(X, y_{d}\right)+G\left(X, y_{d}\right) w=F\left(X, y_{d}\right)+G\left(X, y_{d}\right) \grave{M} \grave{w} ; \quad X(0)=X_{0}
$$

where $F$ and $G$ are smooth mappings of $\mathcal{D} \times \mathbb{R}$, respectively; and the initial condition

$$
X_{0} \in \mathcal{D}_{0}:=\left\{X_{0} \in \mathcal{D} \mid \theta \in \Theta, \check{\theta}_{0} \in \Theta, \Sigma(0)=\gamma^{-2} Q_{0}^{-1}>0, s_{\Sigma}(0)=\gamma^{2} \operatorname{Tr}\left(Q_{0}\right) \leq K_{c}\right\}
$$

Since (29) holds, by Lemma 7 in Pan and Başar (2000), the value function $W$ satisfies a HamiltonJacobi-Isaacs equation.

$$
\begin{aligned}
\frac{\partial W}{\partial X}(X) F\left(X, y_{d}\right) & +\frac{1}{4 \gamma^{2}} \frac{\partial W}{\partial X}(X) G\left(X, y_{d}\right)\left(G\left(X, y_{d}\right)\right)^{\prime}\left(\frac{\partial W}{\partial X}(X)\right)^{\prime} \\
& +Q\left(X, y_{d}\right)=0 ; \quad \forall X \in \mathcal{D}, \forall y_{d} \in \mathbb{R}
\end{aligned}
$$

\footnotetext{
${ }^{4}$ See Appendix B for derivations.
} 
where $Q: \mathcal{D} \times \mathbb{R} \rightarrow \mathbb{R}$ is smooth and given by

$$
Q\left(X, y_{d}\right)=\left|C x+\left(b_{p 0}+\bar{C}_{1} \theta\right) \mu\left(\check{\theta}, \check{x}, y_{d}\right)-y_{d}\right|^{2}+|\xi-\check{\xi}|_{\bar{Q}\left(\Phi, \mu\left(\check{\theta}, \check{x}, y_{d}\right), s_{\Sigma}\right)}^{2}-2(\theta-\check{\theta})^{\prime} P_{r}(\check{\theta})
$$

The closed-loop adaptive system possesses a strong robustness property, which will be stated precisely in the following theorem.

Theorem 1 Consider the robust adaptive control problem formulated in Section 3 with Assumptions 1-7 holding. The robust adaptive controller $\mu$ defined by (28a), with the optimal choice (28b) for $\hat{\xi}$, achieves the following strong robustness properties for the closed-loop system.

1. Given $c_{w} \geq 0$, and $c_{d} \geq 0$, there exists a constant $c_{c} \geq 0$ and a compact set $\Theta_{c} \subset \Theta_{o}$, such that for any uncertainty $\left(x_{0}, \theta, \grave{w}_{[0, \infty)}, y_{d[0, \infty)}\right) \in \mathcal{W}$ with

$$
\left|x_{0}\right| \leq c_{w} ;\left|y_{d}(t)\right| \leq c_{d} ;|\grave{w}(t)| \leq c_{w} ; \quad \forall t \in[0, \infty)
$$

all closed-loop state variables $x, \check{x}, \check{\theta}, \Sigma, s_{\Sigma}$, and $\Phi$ are bounded as follows, $\forall t \in[0, \infty)$,

$$
\begin{array}{r}
|x(t)| \leq c_{c} ;|\check{x}(t)| \leq c_{c} ; \check{\theta}(t) \in \Theta_{c} ;|\overrightarrow{\Phi(t)}| \leq c_{c} \\
K_{c}^{-1} I_{\sigma} \leq \Sigma(t) \leq \gamma^{-2} Q_{0}^{-1} ; \gamma^{2} \operatorname{Tr}\left(Q_{0}\right) \leq s_{\Sigma}(t) \leq K_{c}
\end{array}
$$

Therefore, there is a compact set $S \subseteq \mathcal{D}$ such that $X(t) \in S \forall t \in[0, \infty)$. Hence, there exists a constant $c_{u} \geq 0$ such that $|u(t)| \leq c_{u},|\hat{\xi}(t)| \leq c_{u},|\eta(t)| \leq c_{u},|\lambda(t)| \leq c_{u}$, and $\left|\lambda_{o}(t)\right| \leq c_{u}$.

2. The controller $\mu \in \mathcal{M}_{u}$ achieves disturbance attenuation level $\gamma$ for any uncertainty $\left(x_{0}, \theta\right.$, $\left.\grave{w}_{[0, \infty)}, y_{d[0, \infty)}\right) \in \grave{\mathcal{W}}$.

3. For any uncertainty $\left(x_{0}, \theta, \grave{w}_{[0, \infty)}, y_{d[0, \infty)}\right) \in \mathcal{\mathcal { W }}$ with $\grave{w}_{[0, \infty)} \in \mathcal{L}_{2} \cap \mathcal{L}_{\infty}, y_{d[0, \infty)} \in \mathcal{L}_{\infty}$, and $y_{d[0, \infty)}$ being uniformly continuous, the output of the system, $C x+\left(\bar{C}_{1} \theta+b_{p 0}\right) u$, asymptotically tracks the reference trajectory, $y_{d}$, i.e.,

$$
\lim _{t \rightarrow+\infty}\left(C x(t)+\left(\bar{C}_{1} \theta+b_{p 0}\right) u(t)-y_{d}(t)\right)=0
$$

Proof For the first statement, fix $c_{w} \geq 0$ and $c_{d} \geq 0$, consider any uncertainty $\left(x_{0}, \theta, \grave{w}_{[0, \infty)}\right.$, $\left.y_{d[0, \infty)}\right) \in \mathcal{W}$ that satisfies

$$
\left|x_{0}\right| \leq c_{w} ; \quad|\grave{w}(t)| \leq c_{w} ; \quad\left|y_{d}(t)\right| \leq c_{d} ; \quad \forall t \in[0, \infty)
$$

We define $\left[0, T_{f}\right)$ to be the maximal length interval on which the close-loop system (32) has a solution that lies in $\mathcal{D}$. By Lemma 1, we have $\Sigma$ and $s_{\Sigma}$ are upper and lower bounded as desired on $\left[0, T_{f}\right)$.

Introduce the vector of variables

$$
X_{e}:=\left[\check{\theta}^{\prime} \quad(\tilde{x}-\Phi \tilde{\theta})^{\prime}\right]^{\prime}
$$


and two nonnegative and continuous functions defined on $\mathbb{R}^{n+\sigma}$

$$
\begin{aligned}
U_{M}\left(X_{e}\right) & :=K_{c}|\tilde{\theta}|^{2}+\gamma^{2}|\tilde{x}-\Phi \tilde{\theta}|_{\Pi^{-1}}^{2} \\
U_{m}\left(X_{e}\right) & :=\gamma^{2}|\tilde{\theta}|_{Q_{0}}^{2}+\gamma^{2}|\tilde{x}-\Phi \tilde{\theta}|_{\Pi^{-1}}^{2}
\end{aligned}
$$

then, we have, if we interpret $W$ as $\bar{W}\left(t, X_{e}\right)$,

$$
U_{m}\left(X_{e}\right) \leq \bar{W}\left(t, X_{e}\right) \leq U_{M}\left(X_{e}\right), \quad \forall\left(t, X_{e}\right) \in\left[0, T_{f}\right) \times \mathbb{R}^{n+\sigma}
$$

Since $U_{m}\left(X_{e}\right)$ is continuous, nonnegative and radially unbounded, then $\forall \alpha \in \mathbb{R}$, the set $S_{1 \alpha}:=$ $\left\{X_{e} \in \mathbb{R}^{n+\sigma}: \quad U_{m}\left(X_{e}\right) \leq \alpha\right\}$ is compact or empty. Since $|\grave{w}(t)| \leq c_{w}, \forall t \in[0, \infty)$, we have the following inequality for the derivative of $W$ :

$$
\begin{aligned}
\dot{W}= & -\left|C x+\left(\bar{C}_{1} \theta+b_{p 0}\right) u-y_{d}\right|^{2}-\gamma^{4}|x-\hat{x}-\Phi(\theta-\hat{\theta})|_{\Pi^{-1} \Delta \Pi^{-1}}^{2} \\
& -\epsilon\left(\gamma^{2} \zeta^{2}-1\right)|\theta-\hat{\theta}|_{\left(u \bar{C}_{1}+C \Phi\right)^{\prime}\left(u \bar{C}_{1}+C \Phi\right)}^{2}+2(\theta-\check{\theta})^{\prime} P_{r}(\check{\theta})+\gamma^{2}|w|^{2}-\gamma^{2}\left|w-w_{o p t}\right|^{2} \\
\leq & -\gamma^{4}|x-\check{x}-\Phi(\theta-\check{\theta})|_{\Pi^{-1} \Delta \Pi^{-1}}^{2}+2(\theta-\check{\theta})^{\prime} P_{r}(\check{\theta})+\gamma^{2}\|\grave{M}\|_{2}^{2} c_{w}^{2}
\end{aligned}
$$

Since $-\gamma^{4}|x-\check{x}-\Phi(\theta-\check{\theta})|_{\Pi^{-1} \Delta \Pi^{-1}}^{2}+2(\theta-\check{\theta})^{\prime} P_{r}(\check{\theta})$ will tend to $-\infty$ when $X_{e}$ approaches the boundary of $\Theta_{o} \times \mathbb{R}^{n}$, then there exists a compact set $\Omega_{1}\left(c_{w}\right) \subset \Theta_{o} \times \mathbb{R}^{n}$, such that $\dot{W}<0$ for $\forall X_{e} \in\left(\Theta_{o} \times \mathbb{R}^{n}\right) \backslash \Omega_{1}$. To apply the Lemma 9 in Pan and Başar (2000), we make the following substitutions,

$$
\begin{gathered}
U_{m} \rightarrow W_{1} ; \quad U_{M} \rightarrow W_{2} ; \quad \bar{W} \rightarrow V ; \quad S_{1 \alpha} \rightarrow S_{1 \alpha} ; \quad \mathbb{R}^{n+\sigma} \rightarrow D ; \quad c_{1} \rightarrow \eta \\
S_{1 c_{1}} \rightarrow S_{1 \eta} ; \quad X_{e} \rightarrow \xi ; \quad\left[0, T_{f}\right) \rightarrow\left[t_{0}, t_{1}\right) ; \quad \Omega_{1} \rightarrow K ; \quad n+\sigma \rightarrow n
\end{gathered}
$$

Note that $X_{e}(t) \in \Theta_{o} \times \mathbb{R}^{n}, \forall t \in\left[0, T_{f}\right)$. Then, $\bar{W}\left(t, X_{e}(t)\right) \leq c_{1}$, and $X_{e}(t)$ belongs to a compact set $S_{1 c_{1}} \subseteq \mathbb{R}^{n+\sigma}, \forall t \in\left[0, T_{f}\right)$, for some $c_{1} \in \mathbb{R}$. It follows that the signal $X_{e}$ is bounded, namely, $\tilde{\theta}$ and $\tilde{x}-\Phi \tilde{\theta}$ are bounded.

Based on the derivative of $\tilde{x}-\Phi \tilde{\theta},{ }^{5}$ there is particular linear combination of the components of $\tilde{x}-\Phi \tilde{\theta}$, denoted by $\eta_{L}$,

$$
\begin{aligned}
\frac{\mathrm{d}(\tilde{x}(t)-\Phi(t) \tilde{\theta}(t))}{\mathrm{d} t}= & A_{f}(\tilde{x}(t)-\Phi(t) \tilde{\theta}(t))-\gamma^{-2} \Pi C^{\prime} y(t)+\gamma^{-2} \Pi C^{\prime} y_{d}(t)+\left(D+\gamma^{-2} \Pi C^{\prime} E\right. \\
& \left.-\zeta^{2}\left(\Pi C^{\prime}+L\right) E\right) \grave{M} \grave{w}(t) \\
\eta_{L}= & T_{L}(\tilde{x}-\Phi \tilde{\theta})
\end{aligned}
$$

which is strictly minimum phase and has relative degree 1 with respect to $y$. Then by Lemma 8 , the composite system of (1) and (36) has relative degree 1 from input $u$ to output $\eta_{L}$; and it may serve as a reference systems in the application of Lemma 11 in Pan and Başar (2000) in the following proof.

By Lemma 7, the system (36) with input $y$ and output $\eta_{L}$ may serve as reference system in the application of Lemma 11 of Pan and Başar (2000).

Based on the dynamics of $\eta$, the relative degree for each element of $\eta$ is at least 1 with respect to the input $y$. Taking $\eta_{L}$ as the output and $y$ as the input of the reference system, we conclude $\eta$

\footnotetext{
${ }^{5}$ See Appendix B for detailed derivations.
} 
is bounded by Lemma 11 of Pan and Başar (2000). Based on the dynamics of $\lambda$, the relative degree for each element of $\lambda$ is at least 1 with respect to the input $u$. Taking $\eta_{L}$ as the output and $u$ as the input of the reference system, we conclude $\lambda$ is also bounded.

Based on the dynamics of $\lambda_{o}(23 \mathrm{c})$, we have $\lambda_{o}$ is bounded. Based on the formula for $\Phi,(23 \mathrm{~d})$, we have $\Phi$ is bounded. Since $\tilde{x}-\Phi \tilde{\theta}, \Phi$, and $\tilde{\theta}$ are bounded, we conclude that $\tilde{x}$ is bounded. Define the following equations to separate $x$ into two part:

$$
\begin{aligned}
x & =x_{u}+x_{y} \\
\dot{x}_{u} & =A_{f} x_{u}+\left(B+\bar{A}_{212} \theta-\left(\bar{C}_{1} \theta+b_{p 0}\right)\left(\zeta^{2} L+\Pi C^{\prime}\left(\zeta^{2}-\frac{1}{\gamma^{2}}\right)\right)\right) u \\
\dot{x}_{y} & =A_{f} x_{y}+\left(\bar{A}_{211} \theta+\zeta^{2} L+\Pi C^{\prime}\left(\zeta^{2}-\frac{1}{\gamma^{2}}\right)\right) y+\left(D-\left(\zeta^{2} L+\Pi C^{\prime}\left(\zeta^{2}-\frac{1}{\gamma^{2}}\right)\right) E\right) \grave{M} \grave{w}
\end{aligned}
$$

We observe that the relative degree for each element of $x_{u}$ is at least 1 with respect to the input $u$, and the relative degree for each element of $x_{y}$ is at least 1 with respect to the input $y$. Taking $\eta_{L}$ as the output and $u$ as the input of the reference system, we conclude $x_{u}$ is bounded by Lemma 11 in Pan and Başar (2000). Similarly, Taking $\eta_{L}$ as the output and $y$ as the input of the reference system, we obtain that $x_{y}$ is bounded. Then, we have $x$ is bounded. Further, we have $\check{x}$ is bounded, because $x$ and $\tilde{x}$ are bounded and $\check{x}=x-\tilde{x}$. Since $\check{b}_{0}$ is bounded, and bounded away from 0 , we conclude that $u$ is bounded.

Next, we need to prove the existence of a compact set $\Theta_{c} \subset \Theta_{o}$ such that $\check{\theta}(t) \in \Theta_{c}, \forall t \in\left[0, T_{f}\right)$. First introduce the function $\Upsilon:\left[0, T_{f}\right) \times\left(\Theta_{o} \times \mathbb{R}^{n}\right) \rightarrow \mathbb{R}$ by

$$
\Upsilon\left(t, X_{e}\right):=\bar{W}\left(t, X_{e}\right)+\left(\rho_{o}-P(\check{\theta})\right)^{-1} P(\check{\theta})
$$

We notice that, when $\check{\theta}$ approaches the boundary of $\Theta_{o}, P(\check{\theta})$ approaches $\rho_{o}$. Then $\Upsilon$ approaches $\infty$ as $X_{e}$ approaches the boundary of $\Theta_{o} \times \mathbb{R}^{n}$. We introduce two nonnegative and continuous functions defined on $\Theta_{o} \times \mathbb{R}^{n}$ :

$$
\begin{aligned}
& \Upsilon_{M}\left(X_{e}\right):=U_{M}\left(X_{e}\right)+\left(\rho_{o}-P(\check{\theta})\right)^{-1} P(\check{\theta}) \\
& \Upsilon_{m}\left(X_{e}\right):=U_{m}\left(X_{e}\right)+\left(\rho_{o}-P(\check{\theta})\right)^{-1} P(\check{\theta})
\end{aligned}
$$

Then, by the previous analysis, we have

$$
\Upsilon_{m}\left(X_{e}\right) \leq \Upsilon\left(t, X_{e}\right) \leq \Upsilon_{M}\left(X_{e}\right), \quad \forall\left(t, X_{e}\right) \in\left[0, T_{f}\right) \times\left(\Theta_{o} \times \mathbb{R}^{n}\right)
$$

Note that the set $S_{2 \alpha}:=\left\{X_{e} \in \Theta_{o} \times \mathbb{R}^{n} \mid \Upsilon_{m}\left(X_{e}\right) \leq \alpha\right\}$ is a compact set or an empty set, $\forall \alpha \in \mathbb{R}$. Then, we consider the derivative of $\Upsilon$,

$$
\begin{aligned}
\dot{\Upsilon}= & \dot{\bar{W}}+\rho_{o}\left(\rho_{o}-P(\check{\theta})\right)^{-2} \frac{\partial P}{\partial \theta}(\check{\theta}) \dot{\check{\theta}}(t) \\
\leq & -\left|C x+\left(\bar{C}_{1} \theta+b_{p 0}\right) u-y_{d}\right|^{2}-\gamma^{4}|x-\hat{x}-\Phi(\theta-\hat{\theta})|_{\Pi^{-1} \Delta \Pi-1}^{2}+2(\theta-\check{\theta})^{\prime} P_{r}(\check{\theta}) \\
& -\epsilon\left(\gamma^{2} \zeta^{2}-1\right)|\theta-\hat{\theta}|_{\left(u \bar{C}_{1}+C \Phi\right)^{\prime}\left(u \bar{C}_{1}+C \Phi\right)}^{2}+\gamma^{2}\|\grave{M}\|_{2}^{2} c_{w}^{2}+\rho_{o}\left(\rho_{o}-P(\check{\theta})\right)^{-2}\left(-\frac{\partial P}{\partial \theta}(\check{\theta}) \Sigma P_{r}(\check{\theta})\right. \\
& -\frac{\partial P}{\partial \theta}(\check{\theta})\left(u \Sigma \bar{C}_{1}^{\prime}+\Sigma \Phi^{\prime} C^{\prime}\right)\left(y_{d}-C \check{x}-\left(\bar{C}_{1} \check{\theta}+b_{p 0}\right) u\right)+\frac{\partial P}{\partial \theta}(\check{\theta}) \gamma^{2} \zeta^{2}\left(u \Sigma \bar{C}_{1}^{\prime}+\Sigma \Phi^{\prime} C^{\prime}\right) \\
& \left.\cdot\left(y-C \check{x}-\left(\bar{C}_{1} \check{\theta}+b_{p 0}\right) u\right)\right)
\end{aligned}
$$


which hold as long as $\Sigma>0$ and $\check{\theta} \in \Theta_{o}$. We observe the following facts.

$$
\begin{aligned}
& y_{d}-C \check{x}-u\left(\bar{C}_{1} \check{\theta}+b_{p 0}\right)=0 \\
& \frac{\partial P}{\partial \theta}(\check{\theta}) \gamma^{2} \zeta^{2}\left(u \Sigma \bar{C}_{1}^{\prime}+\Sigma \Phi^{\prime} C^{\prime}\right)\left(y-C \check{x}-\left(\bar{C}_{1} \check{\theta}+b_{p 0}\right) u\right) \\
& \leq\left|\left(\frac{\partial P}{\partial \theta}(\check{\theta})\right)^{\prime}\left(\rho_{o}-P(\check{\theta})\right)^{-1}\right|^{2}+\left|\frac{\left(\rho_{o}-P(\check{\theta})\right)}{2} \gamma^{2} \zeta^{2}\left(u \Sigma \bar{C}_{1}^{\prime}+\Sigma \Phi^{\prime} C^{\prime}\right)\left(y-C \check{x}-\left(\bar{C}_{1} \check{\theta}+b_{p 0}\right) u\right)\right|^{2}
\end{aligned}
$$

Then,

$$
\begin{aligned}
& \dot{\Upsilon} \leq-\gamma^{4}|x-\hat{x}-\Phi(\theta-\hat{\theta})|_{\Pi^{-1} \Delta \Pi^{-1}}^{2}+2(\theta-\check{\theta})^{\prime} P_{r}(\check{\theta})+\gamma^{2}\|\grave{M}\|_{2}^{2} c_{w}^{2} \\
& +\rho_{o}\left(\rho_{o}-P(\check{\theta})\right)^{-4}\left(-\frac{\partial P}{\partial \theta}(\check{\theta}) \Sigma P_{r}(\check{\theta})\left(\rho_{o}-P(\check{\theta})\right)^{2}+\left|\left(\frac{\partial P}{\partial \theta}(\check{\theta})\right)^{\prime}\right|^{2}\right) \\
& +\frac{\rho_{o}}{4}\left|\gamma^{2} \zeta^{2}\left(u \Sigma \bar{C}_{1}^{\prime}+\Sigma \Phi^{\prime} C^{\prime}\right)\left(y-C \check{x}-\left(\bar{C}_{1} \check{\theta}+b_{p 0}\right) u\right)\right|^{2} \\
& \leq-\gamma^{4}|x-\hat{x}-\Phi(\theta-\hat{\theta})|_{\Pi^{-1} \Delta \Pi^{-1}}^{2}+2(\theta-\check{\theta})^{\prime} P_{r}(\check{\theta})+\gamma^{2}\|\grave{M}\|_{2}^{2} c_{w}^{2} \\
& -\rho_{o}\left|\left(\frac{\partial P}{\partial \theta}(\check{\theta})\right)^{\prime}\right|^{2}\left(\rho_{o}-P(\check{\theta})\right)^{-4}\left(K_{c}^{-1} p_{r}(\check{\theta})\left(\rho_{o}-P(\check{\theta})\right)^{2}-1\right)+c
\end{aligned}
$$

where $c \geq 0$ is a constant, which holds as long as $\Sigma>0$ and $\check{\theta} \in \Theta_{o}$. Since $\dot{\Upsilon}$ will tend to $-\infty$ when $X_{e}$ approaches the boundary of $\Theta_{o} \times \mathbb{R}^{n}$, then there exists a compact set $\Omega_{2}\left(c_{w}\right) \subset \Theta_{o} \times \mathbb{R}^{n}$, such that $\forall X_{e} \in\left(\Theta_{o} \times \mathbb{R}^{n}\right) \backslash \Omega_{2}, \dot{\Upsilon}<0$. To use the Lemma 9 in Pan and Başar (2000), we make the following substitutions,

$$
\begin{gathered}
\Upsilon_{m} \rightarrow W_{1} ; \quad \Upsilon_{M} \rightarrow W_{2} ; \quad \Upsilon \rightarrow V ; \quad S_{2 \alpha} \rightarrow S_{1 \alpha} ; \quad \Theta_{o} \times \mathbb{R}^{n} \rightarrow D ; \quad c_{2} \rightarrow \eta \\
S_{2 c_{2}} \rightarrow S_{1 \eta} ; \quad X_{e} \rightarrow \xi ; \quad\left[0, T_{f}\right) \rightarrow\left[t_{0}, t_{1}\right) ; \quad \Omega_{2} \rightarrow K ; \quad n+\sigma \rightarrow n
\end{gathered}
$$

Then, $\Upsilon\left(t, X_{e}(t)\right) \leq c_{2}$, and $X_{e}(t)$ belongs to the compact set $S_{2 c_{2}} \subset \Theta_{o} \times \mathbb{R}^{n}, \forall t \in\left[0, T_{f}\right)$, for some $c_{2} \in \mathbb{R}$. Moreover, there exists a compact set $\Theta_{c} \subset \Theta_{o}$, such that $\check{\theta}(t) \in \Theta_{c}, \forall t \in\left[0, T_{f}\right)$.

Then we can conclude that there exists a compact set $S \subset \mathcal{D}$, such that $X(t) \in S, \forall t \in\left[0, T_{f}\right)$. Therefore, it follows that $T_{f}=\infty$ and $X(t) \in S, \forall t \in[0, \infty)$.

For the second statement, we fix any uncertainty $\left(x_{0}, \theta, \grave{w}_{[0, \infty)}, y_{d[0, \infty)}\right) \in \mathcal{W}$. Since $y_{d}$ and $\grave{w}$ are continuous functions, then, for any $t_{f}>0$, there exist constants $c_{d} \geq 0$ and $c_{w} \geq 0$ such that $\left|y_{d}(t)\right| \leq c_{d}$ and $|\grave{w}(t)| \leq c_{w}, \forall t \in\left[0, t_{f}\right]$. By the first statement, there exists a solution $X:\left[0, t_{f}\right] \rightarrow$ $\mathcal{D}$ for the closed-loop system (32). Then, by causality of system (32) and its smoothness, it implies that the closed-loop system $(32)$ admits a unique solution on $[0, \infty)$. We set

$$
\begin{aligned}
l\left(t, \theta, x, y_{[0, t]}, y_{d[0, t]}\right):= & \gamma^{4}|x-\hat{x}(t)-\Phi(t)(\theta-\hat{\theta}(t))|_{\Pi^{-1} \Delta \Pi^{-1}}^{2}-2(\theta-\check{\theta}(t))^{\prime} P_{r}(\check{\theta}(t)) \\
& +\epsilon\left(\gamma^{2} \zeta^{2}-1\right)|\theta-\hat{\theta}(t)|_{\left(u(t) \bar{C}_{1}+C \Phi(t)\right)^{\prime}\left(u(t) \bar{C}_{1}+C \Phi(t)\right)}^{2}
\end{aligned}
$$

Then, we have

$$
\sup _{\left(x_{0}, \theta, \grave{w}_{[0, \infty)}, y_{d[0, \infty)}\right) \in \mathcal{W}}\left\{\int _ { 0 } ^ { t _ { f } } \left(\left(C x(\tau)+\left(\bar{C}_{1} \theta+b_{p 0}\right) u(\tau)-y_{d}(\tau)\right)^{2}+l\left(\tau, \theta, x(\tau), y_{[0, \tau]}, y_{d[0, \tau]}\right)\right.\right.
$$




$$
\begin{aligned}
& \left.\left.-\gamma^{2}|w(\tau)|^{2}\right) d \tau-\gamma^{2}\left|\left[\begin{array}{ll}
\theta^{\prime}-\check{\theta}_{0}^{\prime} & x_{0}^{\prime}-\check{x}_{0}^{\prime}
\end{array}\right]^{\prime}\right|_{\bar{Q}_{0}}^{2}\right\} \\
& \leq \sup _{\left(x_{0}, \theta, \grave{w}_{[0, \infty)}, y_{d[0, \infty)}\right) \in \grave{W}}\left\{\int _ { 0 } ^ { t _ { f } } \left(\left(C x(\tau)+\left(\bar{C}_{1} \theta+b_{p 0}\right) u(\tau)-y_{d}(\tau)\right)^{2}+l\left(\tau, \theta, x(\tau), y_{[0, \tau]}, y_{d[0, \tau]}\right)\right.\right. \\
& \left.\left.-\gamma^{2}|w(\tau)|^{2}\right) d \tau-\gamma^{2}\left|\left[\begin{array}{ll}
\theta^{\prime}-\check{\theta}_{0}^{\prime} & x_{0}^{\prime}-\check{x}_{0}^{\prime}
\end{array}\right]^{\prime}\right|_{\bar{Q}_{0}}^{2}+\int_{0}^{t_{f}} \dot{W} d \tau-W\left(X\left(t_{f}\right)\right)+W(X(0))\right\} \\
& \leq-W\left(X\left(t_{f}\right)\right) \leq 0
\end{aligned}
$$

This establishes the second statement.

For the last statement, we consider the following inequality,

$$
\int_{0}^{t_{f}} \dot{W} d \tau \leq \int_{0}^{t_{f}}\left(-\left|C x(\tau)+\left(\bar{C}_{1} \theta+b_{p 0}\right) u(\tau)-y_{d}(\tau)\right|^{2}+\gamma^{2}|\grave{M} \grave{w}(\tau)|^{2}\right) d \tau ; \quad \forall t_{f} \geq 0
$$

Then,

$$
\int_{0}^{\infty}\left|C x(\tau)+\left(\bar{C}_{1} \theta+b_{p 0}\right) u(\tau)-y_{d}(\tau)\right|^{2} d \tau \leq \int_{0}^{\infty}\left(\gamma^{2}|\grave{M} \grave{w}(\tau)|^{2}\right) d \tau+W(X(0))<+\infty
$$

By Lemma 2, $X$ is uniformly continuous. Since $y_{d}-C \check{x}$ is uniformly continuous and bounded, $\bar{C}_{1} \check{\theta}+b_{p 0}$ is uniformly continuous and bounded away from 0 , then $u$ is uniformly continuous by Lemmas 3 and 4 . Then, $C x+\left(\bar{C}_{1} \theta+b_{p 0}\right) u-y_{d}$ is uniformly continuous. By Lemma 5 , we have

$$
\lim _{t \rightarrow+\infty}\left(C x(t)+\left(\bar{C}_{1} \theta+b_{p 0}\right) u(t)-y_{d}(t)\right)=0
$$

This completes the proof of the theorem.

\section{Example}

In this section, we present one example to illustrate the main results of this paper. The design was carried out using MATLAB ${ }^{\mathrm{TM}}$ symbolic computation tools, and the closed-loop system was simulated using SIMULINK ${ }^{\mathrm{TM}}$.

Consider the following circuit problem in Figure 1 , where $v_{i}$ is the input voltage source; $v_{o}$ is the measured output; $v_{e}$ is an unknown sinusoidal voltage source; $v_{w}$ is an unmeasured exogenous voltage source. The objective is to achieve asymptotic tracking of $v_{o}-v_{w}$ to the reference trajectory $y_{d}$.

The equations that describe the circuit are obtained as

$$
y=v_{e}+b_{0} u+\grave{w}
$$

where $u=v_{i}, y=v_{o}, \grave{w}=v_{w}$, and $b_{0}$ is the ratio of $v_{t}$ to $v_{i}$, which is assumed to be unknown and belong to the interval $[0.1,1]$, whose true value is set to 1 for illustration purposes. $v_{e}$ can be modeled as the output of a second-order linear system as the following,

$$
\begin{aligned}
& \dot{\grave{x}}_{1}=\grave{x}_{2} ; \quad \grave{x}_{1}(0)=1 \\
& \dot{\grave{x}}_{2}=\bar{\theta}_{2} \grave{x}_{1} ; \quad \grave{x}_{2}(0)=1
\end{aligned}
$$




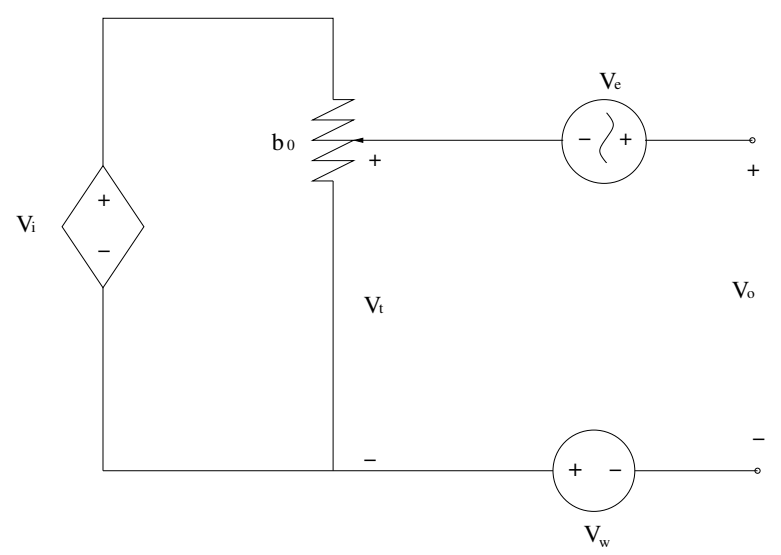

Figure 1: Diagram of the circuit.

where $\bar{\theta}_{2}$ is assumed to be unknown and belong to the interval $[-4,0]$, whose true value is set to -4 for illustration purposes, and the initial conditions are set for illustration purposes. Note that the true system satisfies the Assumptions 1 and 2 .

To normalize the parameters, we set $b_{p 0}=0.55$, and define $\theta=\left[\begin{array}{ll}\theta_{1} & \theta_{2} \\ \theta_{3}\end{array}\right]^{\prime}$, where $\theta_{1}=\left(b_{0}-\right.$ $\left.b_{p 0}\right) / 0.45, \theta_{2}=\left(\bar{\theta}_{2}+2\right) / 2$ and $\theta_{3}=\theta_{1} \theta_{2}$. Then, the true value for parameter vector $\theta$ is $[1-1-1]^{\prime}$, and $\theta_{1}, \theta_{2}$, and $\theta_{3}$ all belong to the interval $[-1,1]$.

Introduce the state transformation $x=\grave{x}$, and disturbance transformations, $w=\left[\begin{array}{cc}1 & \theta_{2} / 3\end{array}\right]^{\prime} \grave{w}$, we obtain the design model for the adaptive controller

$$
\begin{aligned}
\dot{x} & =\left[\begin{array}{cc}
0 & 1 \\
-2 & 0
\end{array}\right] x+\left(y\left[\begin{array}{lll}
0 & 0 & 0 \\
0 & 2 & 0
\end{array}\right]+u\left[\begin{array}{ccc}
0 & 0 & 0 \\
0 & -1.1 & -0.9
\end{array}\right]\right) \theta+\left[\begin{array}{cc}
0 & 0 \\
0 & -6
\end{array}\right] w \\
y & =\left[\begin{array}{ll}
1 & 0
\end{array}\right] x+0.55 u+\left[\begin{array}{lll}
0.45 & 0 & 0
\end{array}\right] \theta u+\left[\begin{array}{ll}
1 & 0
\end{array}\right] w
\end{aligned}
$$

For the adaptive control design, the ultimate lower bound for the achievable disturbance attenuation level is 1 with respect to $w$. We set the desired disturbance attenuation level $\gamma=10$.

The convex function $P(\theta)$ is chosen as

$$
P(\theta)=0.8 \theta_{1}^{2}+0.1\left(\theta_{2}^{2}+\theta_{3}^{2}\right)
$$

For other design and simulation parameters, we select

$$
\begin{gathered}
\check{x}_{0}=\left[\begin{array}{cc}
0 & 0
\end{array}\right]^{\prime} ; \check{\theta}_{0}=\left[\begin{array}{ccc}
0 & 0 & 0
\end{array}\right]^{\prime} ; Q_{0}=0.005 I_{3} ; \Delta_{1}=I_{2} ; p_{n}=\left[\begin{array}{cc}
0 & 1
\end{array}\right]^{\prime} \\
\Phi_{0}=\mathbf{0}_{2 \times 3} ; \quad K_{c}=1.5 ; \quad \rho_{o}=1.15 ; \quad \beta_{\Delta}=0 ; \epsilon=K_{c}^{-1} s_{\Sigma} ; \eta_{0}=\mathbf{0}_{2 \times 1} ; \quad \lambda_{0}=\mathbf{0}_{2 \times 1}
\end{gathered}
$$

Then, we obtain

$$
\Pi=\left[\begin{array}{cc}
10.998 & 9.873 \\
9.873 & 129.49
\end{array}\right]
$$

Set the reference trajectory, $y_{d}=\sqrt{|\sin (3 t)|}$, which is uniformly continuous on $[0, \infty)$. We present two sets of simulation results for this example. The first set is to illustrate the regulatory 
behaviour of the adaptive controller. We set $\grave{w}(t) \equiv 0$. The result are shown in Figure 2 . We observe that the parameter estimates converge to their true values, and the tracking error converges to 0. The transient of the system is well-behaved, and the control magnitude is upper bounded by 2.2. The convergence rate is clearly exponential. When $t=175$, the magnitude of the tracking error reduces to $10^{-14}$, and magnitude of the parameter estimation error reduces to $10^{-13}$.

The second set of simulation results is to illustrate the robustness property of the adaptive controller. We set $\grave{w}(t)=0.4 \sin (t)$. The simulation results are shown in the Figure 3 . We observe that the parameter estimates no longer converge to the true values, but the output tracking error satisfies the targeted attenuation level as desired according to Figure 3(f). The transient of the system is well-behaved which lasted about 5 seconds, and the control magnitude is upper bounded by 2.3 .

\section{Conclusions}

In this paper, we studied the adaptive control design for tracking and disturbance attenuation for SISO linear systems with zero relative degree under noisy output measurements. We assume that the linear system has a known upper bound of the dynamic order, has a strictly minimum phase transfer function with known relative degree 0 . We allow the system to be uncontrollable, as long as the uncontrollable part is stable in the sense of Lyapunov and those uncontrollable modes on the $j \omega$-axis are uncontrollable from the disturbance input. Under these assumptions, the system may be transformed into the design model, which is linear in all of the unknown quantities. The objectives of the control design are to make the noiseless output of the system to asymptotically track the reference trajectory, and guarantee the boundedness of all closed-loop signals, while rejecting the uncertainties in the system, which comprises the initial state of the system, the true value of the unknown parameter vector, the waveform of the exogenous disturbance input, and the reference trajectory. We use $H^{\infty}$-optimal control formulation and game theoretic approach to derive the robust adaptive controller. We treat the unknown parameter vector as part of the expanded state vector, and formulate this adaptive control problem as a nonlinear $H^{\infty}$-optimal control problem with imperfect state measurements. For the design model, we assume that the measurement channel is noisy, such that the estimation step is a nonsingular optimization problem. We further assume that the unknown parameter vector belongs to a convex compact set characterized by a known smooth nonnegative radially unbounded and strictly convex function $P(\theta)$. Furthermore, for any parameter vector belonging to the set, the corresponding high frequency gain is never zero. Then, the cost-to-come function analysis is applied to derive the worst-case identifier and state estimator, which have a finite-dimensional structure. Using a priori information on the parameter vector, a smooth soft projection algorithm is applied in the estimation step, which relieves the persistency of excitation condition for the closed-loop system. Then, the closed-loop system is robust with or without the persistently exciting signals. After the estimation step is completed, the original problem becomes a nonlinear $H^{\infty}$-optimal control problem under full-information measurements. Then, the controller can be obtained directly from the cost function in one step. The controller then achieve the desired disturbance attenuation level, with the ultimate lower bound of the attenuation level being the noise intensity in the measurement channel. It guarantees the boundedness of all closed-loop signals and achieves asymptotic tracking of uniformly continuous bounded reference trajectories when the disturbance is of finite energy and bounded. Because of the assumptions we made on the unknown system, the adaptive controller can asymptotically cancel out the effect 


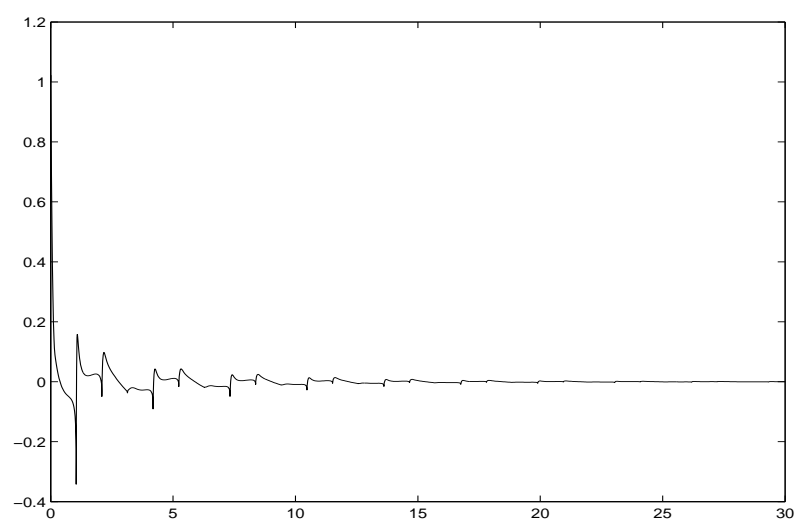

(a)

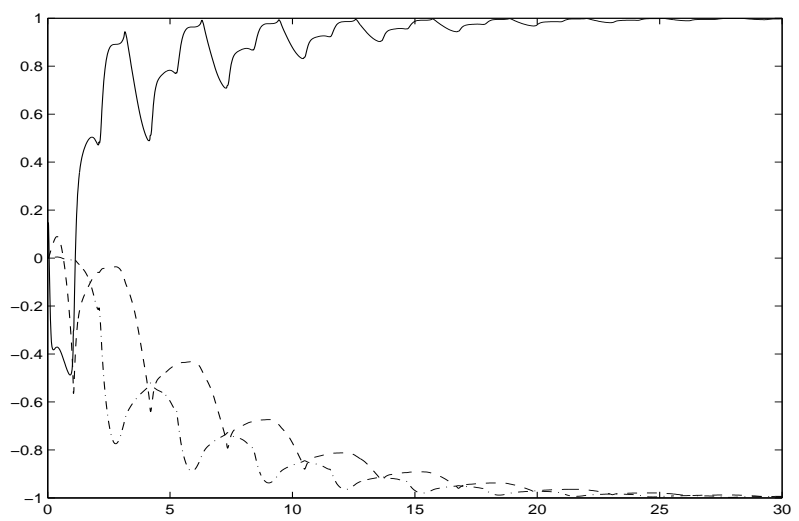

(c)

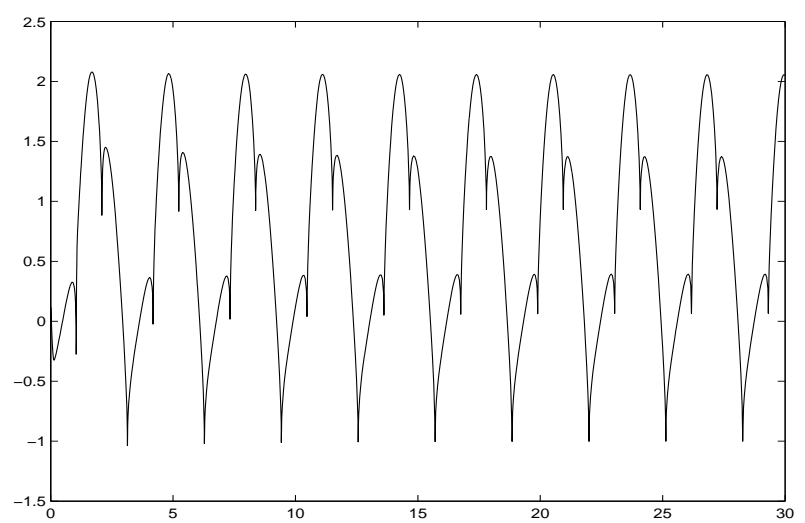

(e)

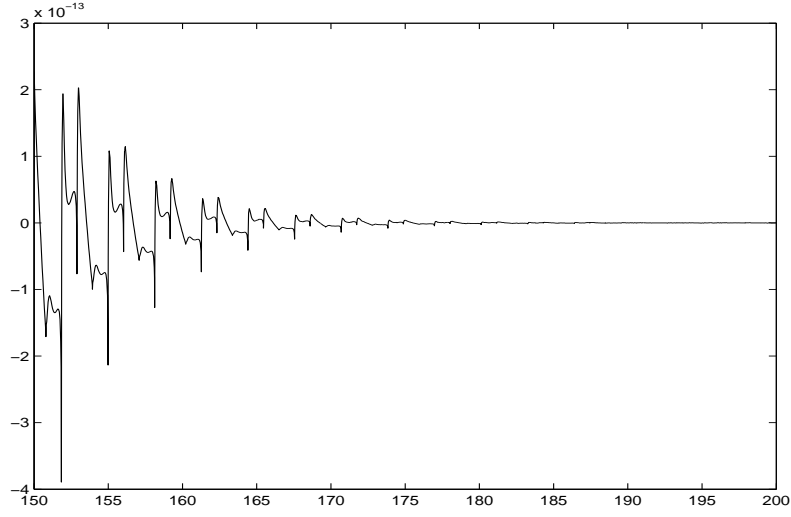

(b)

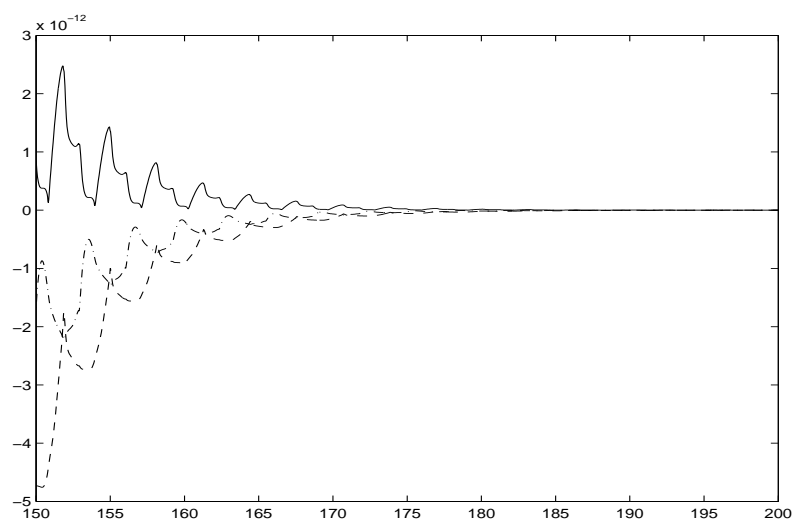

(d)

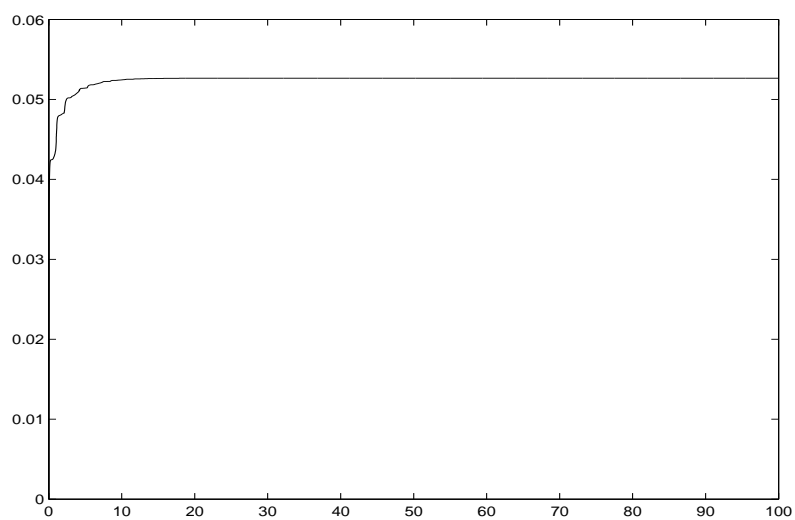

(f)

Figure 2: System response under reference trajectory $y_{d}(t)=\sqrt{|\sin (3 t)|}$ and $\grave{w}(t)=0$.

(a) Tracking error; (b) Tracking error (long term); (c) Parameter estimates; solid line for $\check{\theta}_{1}$; dash line for $\check{\theta}_{2}$; and dash dot line for $\check{\theta}_{3}$; (d) Parameter estimation error (long term); solid line for $\theta_{1}-\check{\theta}_{1}$; dash line for $\theta_{2}-\check{\theta}_{2}$; and dash dot line for $\theta_{3}-\check{\theta}_{3}$; (e) Control input; (f) A performance index $\int_{0}^{t}\left(\left(C x+b_{0} u-y_{d}\right)^{2}-\gamma^{2}|w|^{2}\right) d \tau$. 


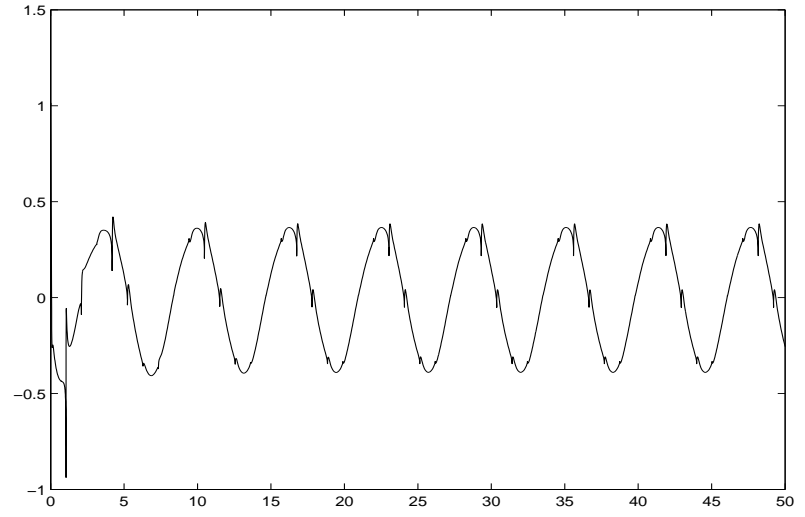

(a)

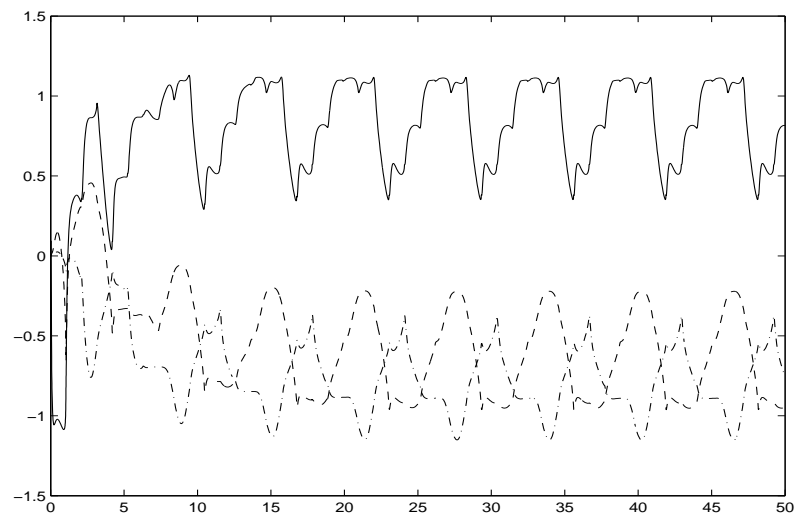

(c)

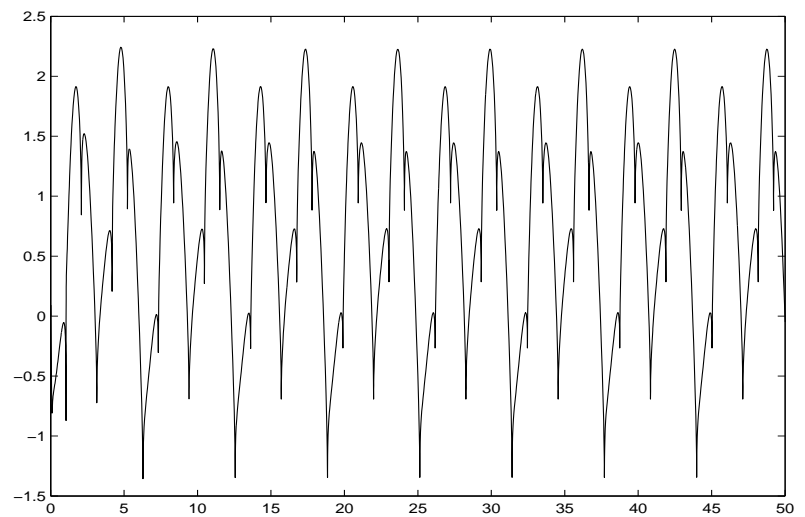

(e)

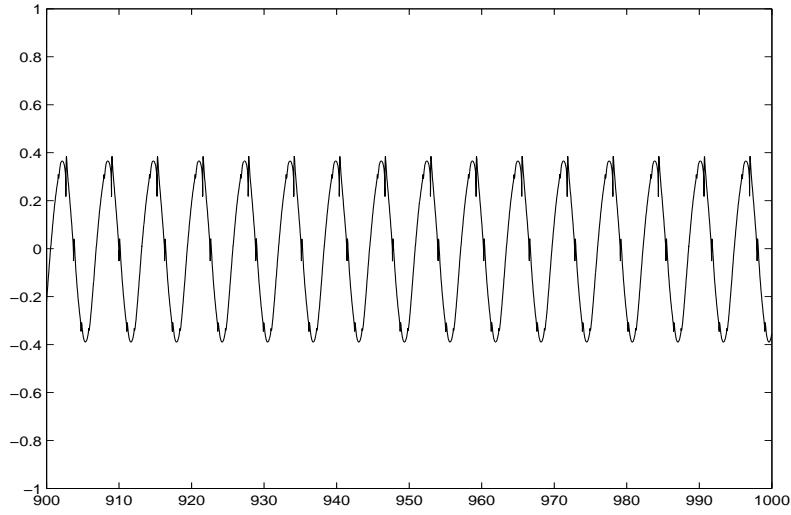

(b)

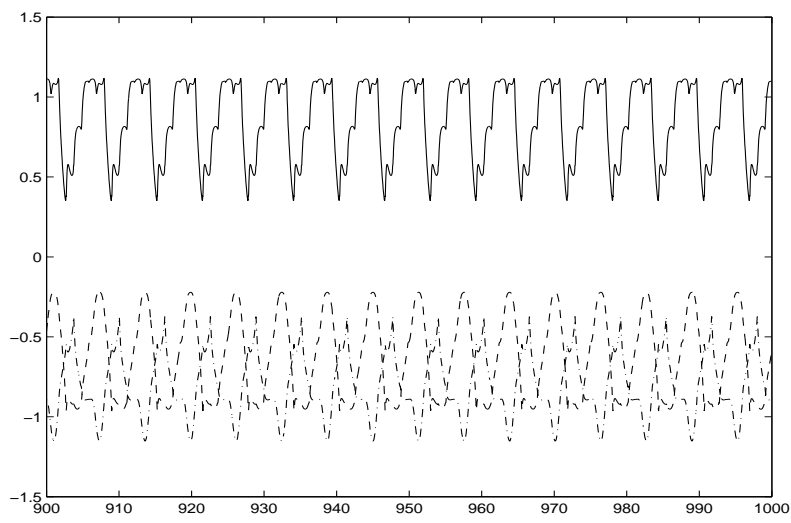

(d)

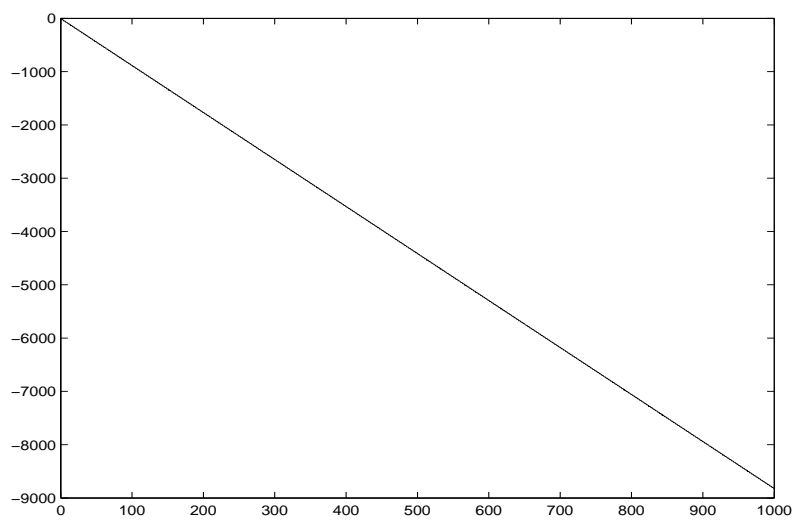

(f)

Figure 3: System response under reference trajectory $y_{d}(t)=\sqrt{|\sin (3 t)|}$ and $\grave{w}(t)=0.4 \sin (t)$. (a) Tracking error; (b) Tracking error (long term); (c) Parameter estimates; solid line for $\check{\theta}_{1}$; dash line for $\check{\theta}_{2}$; and dash dot line for $\check{\theta}_{3}$; (d) Parameter estimates (long term); solid line for $\check{\theta}_{1}$; dash line for $\check{\theta}_{2}$; and dash dot line for $\check{\theta}_{3}$; (e) Control input; (f) A performance index $\int_{0}^{t}\left(\left(C x+b_{0} u-y_{d}\right)^{2}-\right.$ $\left.\gamma^{2}|w|^{2}\right) d \tau$. 
of exogenous sinusoidal inputs with unknown magnitudes, phases, and frequencies, as long as we extend our system model to incorporate the knowledge of the existence of such sinusoidal inputs. This property of our adaptive controller has significant impact on active noise cancellation applications. This feature is illustrated by a numerical example, which corroborates all of our theoretical findings.

Future research directions that are of interest are described as follows. One direction lies in the generalization of the results to nonlinear systems. Another fruitful direction lies in the extension of the results to multiple-input and multiple-output systems. The class of MIMO systems under study involves two subsystems, $\mathbf{S}_{\mathbf{1}}$, and $\mathbf{S}_{\mathbf{2}}$, interconnected to each other, where the connection is serial. Preliminary results have been obtained for this class of MIMO systems.

\section{A Seven Lemmas}

Lemma 2 Assume $f:[0, \infty) \rightarrow \mathbb{R}$ is differentiable in $t$, and satisfies

$$
\left|f^{(1)}(t)\right| \leq M<\infty ; \quad \forall t \in[0, \infty)
$$

for some $M \in \mathbb{R}, M \geq 0$. Then, $f$ is uniformly continuous on $[0, \infty)$.

Proof Given a $\epsilon>0$, we take $\delta(\epsilon)=\frac{\epsilon}{M+1}$. Then, if $x, y \in[0, \infty)$ and $|x-y| \leq \delta(\epsilon)$, from the Mean Value Theorem, we have

$$
|f(x)-f(y)|=\left|f^{(1)}(\xi)\right| \cdot|x-y| \leq M \cdot \delta(\epsilon)=M \cdot \frac{\epsilon}{M+1}<\epsilon
$$

This completes the proof of this Lemma.

Lemma 3 Assume $g:[0, \infty) \rightarrow \mathbb{R}$ and $h:[0, \infty) \rightarrow \mathbb{R}$ are uniformly continuous. If $\exists M>0$, such that $|g(t)| \leq M$ and $|h(t)| \leq M, \forall t \in[0, \infty)$, then $g \cdot h$ is uniformly continuous on $[0, \infty)$.

Proof Since $g$ is uniformly continuous, $\forall \epsilon>0, \exists \delta_{g}(\epsilon)>0$ such that $|g(x)-g(y)|<\epsilon$, $\forall x, y \in[0, \infty)$ with $|x-y|<\delta_{g}(\epsilon)$; since $h$ is uniformly continuous, $\forall \epsilon>0, \exists \delta_{h}(\epsilon)>0$ such that $|h(x)-h(y)|<\epsilon, \forall x, y \in[0, \infty)$ with $|x-y|<\delta_{h}(\epsilon)$. Then, given an $\epsilon>0$, we take $\delta(\epsilon)=\min \left\{\delta_{h}\left(\frac{\epsilon}{2 M}\right), \delta_{g}\left(\frac{\epsilon}{2 M}\right)\right\}$, and we have

$$
\begin{aligned}
|g(x) \cdot h(x)-g(y) \cdot h(y)| & =|g(x) \cdot h(x)-g(x) \cdot h(y)+g(x) \cdot h(y)-g(y) \cdot h(y)| \\
& \leq|g(x)| \cdot|h(x)-h(y)|+|h(y)| \cdot|g(x)-g(y)| \\
& \leq M|h(x)-h(y)|+M|g(x)-g(y)| \\
& <\epsilon ; \quad \forall x, y \in[0, \infty) \text { with }|x-y|<\delta(\epsilon)
\end{aligned}
$$

This completes the proof of this Lemma.

Lemma 4 Assume $h:[0, \infty) \rightarrow \mathbb{R}$ is uniformly continuous. If $\exists M>0$, such that $|h(t)| \geq M$, $\forall t \in[0, \infty)$, then $\frac{1}{h(t)}$ is uniformly continuous on $[0, \infty)$. 
Proof Since $h$ is uniformly continuous, $\forall \epsilon>0, \exists \delta_{h}(\epsilon)>0$ such that $|h(x)-h(y)|<\epsilon, \forall x, y \in$ $[0, \infty)$ with $|x-y|<\delta_{h}(\epsilon)$. Then, given an $\epsilon>0$, we take $\delta(\epsilon)=\delta_{h}\left(\frac{\epsilon}{M^{2}}\right)$, and we have

$$
\begin{aligned}
\left|\frac{1}{h(x)}-\frac{1}{h(y)}\right| & =\left|\frac{h(x)-h(y)}{h(x) \cdot h(y)}\right| \\
& \leq M^{2}|h(x)-h(y)| \\
& <\epsilon ; \quad \forall x, y \in[0, \infty) \text { with }|x-y|<\delta(\epsilon)
\end{aligned}
$$

This completes the proof of this Lemma.

Lemma 5 If $f:[0, \infty) \rightarrow \mathbb{R}$ is uniformly continuous in $t$, and satisfies:

$$
\int_{0}^{\infty}|f(\tau)|^{p} \mathrm{~d} \tau<\infty ; \quad p \in(0, \infty)
$$

then $\lim _{t \rightarrow \infty} f(t)=0$.

Proof We prove it by contradiction. Suppose $\lim _{t \rightarrow \infty} f(t) \neq 0$, then $\exists \epsilon_{0}>0, \forall T>0$ exists $t_{0}>T$ such that $\left|f\left(t_{0}\right)\right|>\epsilon_{0}$.

From the definition of uniformly continuous, take $\epsilon=\frac{\epsilon_{0}}{2}>0$, there exists $\delta>0$ such that $\forall t_{1}$, $t_{2} \in[0, \infty)$ with $\left|t_{1}-t_{2}\right|<\delta$, we have

$$
\left|f\left(t_{1}\right)-f\left(t_{2}\right)\right| \leq \epsilon
$$

For the above $t_{0}$, it follows $|f(t)| \geq \frac{\epsilon_{0}}{2}$ when $t \in\left[t_{0}-\delta, t_{0}+\delta\right] \cap[0, \infty)$.

First we take $T_{1}=\delta$, then we may obtain $t_{1}>T_{1}$ and a time interval $\left[t_{1}-\delta, t_{1}+\delta\right]$, such that $|f(t)| \geq \frac{\epsilon_{0}}{2}, \forall t \in\left[t_{1}-\delta, t_{1}+\delta\right]$. Next, take $T_{2}=t_{1}+2 \delta$, then we obtain a $t_{2}>T_{2}$ and a time interval $\left[t_{2}-\delta, t_{2}+\delta\right]$, such that $|f(t)| \geq \frac{\epsilon_{0}}{2}, \forall t \in\left[t_{2}-\delta, t_{2}+\delta\right]$. Continue such procedure, we obtain a sequence $\left\{t_{n}\right\}_{n=1}^{\infty}$ and corresponding disjoint time intervals $\left[t_{n}-\delta, t_{n}+\delta\right], n=1,2 \cdots$. Then

$$
\begin{aligned}
\int_{0}^{\infty}|f(\tau)|^{p} d \tau & \geq \int_{t_{1}-\delta}^{t_{1}+\delta}|f(\tau)|^{p} d \tau+\int_{t_{2}-\delta}^{t_{2}+\delta}|f(\tau)|^{p} d \tau+\cdots+\int_{t_{n}-\delta}^{t_{n}+\delta}|f(\tau)|^{p} d \tau+\cdots \\
& \geq \int_{t_{1}-\delta}^{t_{1}+\delta}\left|\frac{1}{2} \epsilon_{0}\right|^{p} \mathrm{~d} \tau+\int_{t_{2}-\delta}^{t_{2}+\delta}\left|\frac{1}{2} \epsilon_{0}\right|^{p} \mathrm{~d} \tau+\cdots+\int_{t_{n}-\delta}^{t_{n}+\delta}\left|\frac{1}{2} \epsilon_{0}\right|^{p} \mathrm{~d} \tau+\cdots \\
& \geq\left(\frac{1}{2} \epsilon_{0}\right)^{p} 2 \delta N \quad \forall N \in \mathbb{N}
\end{aligned}
$$

which contradicts $\int_{0}^{\infty}|f(\tau)|^{p} \mathrm{~d} \tau<\infty$.

Therefore $\lim _{t \rightarrow \infty} f(t)=0$.

Lemma 6 Consider the system (1) under Assumption 2. Assume $\grave{n}<n$. Then, we can always add additional $(n-\grave{n})$-dimensional dynamics, such that the expanded system is of order $n$ and satisfies Assumption 2. Furthermore, the expanded system admits the same mapping from $\left(\grave{x}_{0}, u_{[0, \infty)}, \grave{w}_{[0, \infty)}\right)$ to $y_{[0, \infty)}$ as system (1).

Proof We will discuss two exhaustive and mutually exclusive cases: Case 1: $\grave{n}=0$; Case 2 : $\grave{n}>0$. First, consider Case 1: $\grave{n}=0$. We expand the system as following,

$$
\begin{aligned}
& \dot{\tilde{\grave{x}}}=\tilde{\grave{A}}_{22} \tilde{\tilde{x}} ; \quad \tilde{\grave{x}}(0)=0 \\
& y=\tilde{\grave{C}} \tilde{\dot{x}}+b_{0} u+\grave{E} \grave{w}
\end{aligned}
$$


where $\tilde{\grave{x}}$ is the $n$-dimensional state vector; $\tilde{\hat{A}}_{22}$ is Hurwitz, and $\left(\tilde{\hat{A}}_{22}, \tilde{\grave{C}}\right)$ is observable. We notice that, since $(37 \mathrm{a})$ is a stable system with zero initial conditions, the trajectory $\tilde{\grave{x}}$ will always be zeros. Then, the trajectory $y$ will remains the same for the expanded system (37) and the original system (1) when the trajectories for $u$ and $\grave{w}$ remain unchanged. Clearly, (37) satisfies Assumption 2 .

Next, we consider Case 2: $\grave{n}>0$. Since system (1) satisfies Assumption 2, we assume it is given in observer canonical form. Then, we expand the original system to $n$ dimensional by adding the $\tilde{\grave{x}}$ dynamics, which is $(n-\grave{n})$-dimensional,

$$
\begin{aligned}
{\left[\begin{array}{c}
\dot{\grave{x}} \\
\dot{\grave{x}}
\end{array}\right] } & =\left[\begin{array}{cc}
\grave{A} & \tilde{\hat{A}}_{12} \\
\mathbf{0} & \tilde{\grave{A}}_{22}
\end{array}\right]\left[\begin{array}{c}
\grave{x} \\
\check{\grave{x}}
\end{array}\right]+\left[\begin{array}{c}
\grave{B} \\
\mathbf{0}
\end{array}\right] u+\left[\begin{array}{c}
\grave{D} \\
\mathbf{0}
\end{array}\right] \grave{w} ;\left[\begin{array}{c}
\grave{x}(0) \\
\check{\grave{x}}(0)
\end{array}\right]=\left[\begin{array}{c}
\grave{x}_{0} \\
\mathbf{0}
\end{array}\right] \\
y & =\left[\begin{array}{ll}
\grave{C} & \mathbf{0}
\end{array}\right]\left[\begin{array}{c}
\grave{x} \\
\check{\grave{x}}
\end{array}\right]+b_{0} u+\grave{E} \grave{w}
\end{aligned}
$$

where $\tilde{\hat{A}}_{12}=\left[\begin{array}{cc}\mathbf{0} & -\tilde{C}^{\prime}\end{array}\right]^{\prime}, \tilde{\grave{C}}$ is a row vector, $\tilde{\hat{A}}_{22}$ is Hurwitz, and the pair $\left(\tilde{\hat{A}}_{22}, \tilde{\grave{C}}\right)$ is observable.

We observe that the trajectories $\grave{x}$ and $y$ in (38) are as same as those in (1), when $\grave{x}_{0}, u$ and $\grave{w}$ remain unchanged.

To check the observability of (38), fix any $\lambda \in \mathbb{C}$, we observe

$$
\left[\begin{array}{cc}
\grave{C} & \mathbf{0} \\
\lambda I-\grave{A} & -\tilde{\grave{A}}_{12} \\
\mathbf{0} & \lambda I-\tilde{\check{A}}_{22}
\end{array}\right]=\left[\begin{array}{cccc|c}
1 & 0 & \cdots & 0 & \\
* & -1 & \cdots & 0 & \mathbf{0}_{\grave{n} \times(n-\grave{n})} \\
\vdots & \vdots & \ddots & \vdots & \\
* & * & \cdots & -1 & \\
\hline & & * & & \check{C}_{\tilde{\grave{A}}_{22}}
\end{array}\right]
$$

where $*$ stands for arbitrary scalars or matrices. Since the pair $\left(\tilde{\grave{A}}_{22}, \tilde{C}\right)$ is observable, the matrix (39) is of full column rank $\forall \lambda \in \mathbb{C}$. Then, system (38) is observable.

Since the expanded dynamics, $\tilde{x}$, is uncontrollable from $u$, the transfer function of (38) is the same one of (1), and it is strictly minimum phase with relative degree 0 .

To check others conditions of Assumption 2, we follow the derivation below,

First, we transform the system (1) into Jordan canonical form representation. We choose an invertible complex matrix $T_{J}$. The transformation $\grave{x}=T_{J} z$ will transform the dynamics (1) into

$$
\begin{aligned}
& \dot{z}=\left[\begin{array}{lll}
J_{1} & & \\
& \ddots & \\
& & J_{k}
\end{array}\right] z+\left[\begin{array}{c}
B_{J 1} \\
\vdots \\
B_{J k}
\end{array}\right] u+\left[\begin{array}{c}
D_{J 1} \\
\vdots \\
D_{J k}
\end{array}\right] \grave{w} \\
& y=\left[\begin{array}{lll}
C_{J 1} & \cdots & C_{J k}
\end{array}\right] z+b_{0} u+\grave{E} \grave{w}
\end{aligned}
$$

where $J_{i}$ is a Jordan block associated with eigenvalue $\lambda_{i}, i=1, \cdots, k, k \in \mathbb{N}$; in the column vectors of $T_{J}$, complex conjugate pairs appear together. Since a single-output Jordan canonical form state space representation is observable only if there is only one Jordan block associated with each distinct eigenvalue, then $i \neq j \Rightarrow \lambda_{i} \neq \lambda_{j}, \forall i, j \in\{1, \cdots, k\}$. Partition $T_{J}=\left[\begin{array}{lll}T_{J 1} & \cdots & T_{J k}\end{array}\right]$ 
accordingly. For any $i, j \in\{1, \cdots, k\}, \lambda_{i}$ is the complex conjugate of $\lambda_{j}$, implies that $T_{J i}$ is the complex conjugate of $T_{J j}$. By Assumption 2, if $\lambda_{i}$ is any uncontrollable mode from $u$, then the last row of $B_{i}$ is zero; in addition, if $\operatorname{Re}\left(\lambda_{i}\right)=0$, then the last row of $D_{i}$ is zero. When $\operatorname{Re}\left(\lambda_{i}\right)=0$ and $\lambda_{i}$ is an uncontrollable mode, the second to last row of $B_{J i}$ must be nonzero, otherwise, the uncontrollable part of (1) with respect to $u$ will contain a Jordan block associated with $\lambda_{i}$ of order at least 2, which is not stable in the sense of Lyapunov. Next, we separate the above Jordan Canonical form into controllable and uncontrollable parts with respect to $u$, and we denote them as $z_{c}$ and $z_{\bar{c}}$, respectively. Then, we separate the uncontrollable part $z_{\bar{c}}$ into $\operatorname{Re}(\lambda)<0$ and $\operatorname{Re}(\lambda)=0$ parts, and we denote them as $z_{3}$ and $z_{4}$, respectively. This corresponds to the existence of a permutation matrix $T_{c}$, and the transformation $z=T_{c}\left[\begin{array}{llll}z_{c}^{\prime} & z_{3}^{\prime} & z_{4}^{\prime}\end{array}\right]^{\prime}$ will transform (40) into

$$
\begin{aligned}
& {\left[\begin{array}{c}
\dot{z}_{c} \\
\hline \dot{z}_{3} \\
\dot{z}_{4}
\end{array}\right]=\left[\begin{array}{c|cc}
\bar{A}_{c} & \multicolumn{2}{|c}{\bar{A}_{a}} \\
\hline \mathbf{0}_{\left(n-n_{1}\right) \times n_{1}} & \bar{A}_{33} & \mathbf{0} \\
\mathbf{0} & \bar{A}_{44}
\end{array}\right]\left[\begin{array}{c}
z_{c} \\
\hline z_{3} \\
z_{4}
\end{array}\right]+\left[\begin{array}{c}
\bar{B}_{c} \\
\hline \mathbf{0} \\
\mathbf{0}
\end{array}\right] u+\left[\begin{array}{c}
\bar{D}_{c} \\
\hline \bar{D}_{3} \\
\mathbf{0}
\end{array}\right] \grave{w}} \\
& y=\left[\begin{array}{l|ll}
\bar{C}_{c} & \bar{C}_{3} & \bar{C}_{4}
\end{array}\right]\left[\begin{array}{c}
z_{c} \\
z_{3} \\
z_{4}
\end{array}\right]+b_{0} u+\grave{E} \grave{w}
\end{aligned}
$$

where $z_{c}$ is the $n_{1}$-dimensional controllable part; $z_{3}$ and $z_{4}$ are $n_{2}$ - and $n_{3}$-dimensional, respectively, and compose the uncontrollable part; $n_{i} \in \mathbb{N} \cup\{0\}, i=1,2,3$. The matrix $T_{c}$ can be taken such that $\bar{A}_{c}=\left[\begin{array}{ccc}\bar{J}_{c 1} & & \\ & \ddots & \\ & & \bar{J}_{c k_{1}}\end{array}\right], \bar{A}_{33}=\left[\begin{array}{ccc}\bar{J}_{31} & & \\ & \ddots & \\ & & \bar{J}_{3 k_{2}}\end{array}\right]$, and $\bar{A}_{44}=\left[\begin{array}{ccc}\bar{J}_{41} & & \\ & \ddots & \\ & & \bar{J}_{4 k_{3}}\end{array}\right]$, where $0 \leq k_{1}, k_{2}, k_{3} \leq k ; J_{c i}, i=1, \cdots, k_{1}, J_{3 j}, j=1, \cdots, k_{2}, J_{4 l}, l=1, \cdots, k_{3}$, are Jordan blocks associated with eigenvalues $\lambda_{c i}, i=1, \cdots, k_{1}, \lambda_{3 j}, j=1, \cdots, k_{2}, \lambda_{4 l}, l=1, \cdots, k_{3}$, respectively. $\lambda_{c i} \neq \lambda_{c j}$, if $i \neq j ; \lambda_{3 i} \neq \lambda_{3 j}$, if $i \neq j$; and $\lambda_{4 i} \neq \lambda_{4 j}$, if $i \neq j$. Furthermore, $\forall i \in\left\{1, \cdots, k_{3}\right\}$, $\bar{J}_{4 i}$ is of order 1 , and $\lambda_{4 i}$ has zero real part; $\forall i \in\left\{1, \cdots, k_{2}\right\}, \lambda_{3 i}$ has negative real part. Complex conjugate pairs appear together in each of the set $\left\{\lambda_{c 1}, \cdots, \lambda_{c k_{1}}\right\},\left\{\lambda_{31}, \cdots, \lambda_{3 k_{2}}\right\},\left\{\lambda_{41}, \cdots, \lambda_{4 k_{3}}\right\}$. Partition $T_{J} T_{c}=\left[\begin{array}{lll|lll|lll}T_{J c 1} & \cdots & T_{J c k_{1}} \mid T_{J 31} & \cdots & T_{J 3 k_{2}} \mid T_{J 41} & \cdots & T_{J 4 k_{3}}\end{array}\right]$ accordingly. For any $i$, $j \in\left\{1, \cdots, k_{1}\right\}, \lambda_{c i}$ is the complex conjugate of $\lambda_{c j}$, implies $T_{J c i}$ is the complex conjugate of $T_{J c j}$. Similar statements can be made for $\lambda_{3 i}$ and $\lambda_{3 j}$, and also for $\lambda_{4 i}$ and $\lambda_{4 j}$.

It is easy to see that, we can choose three complex invertible matrices $T_{\bar{J} c}, T_{\bar{J} 3}, T_{\bar{J} 4}$, such that $T_{\bar{J}}=$ block diagonal $\left(T_{\bar{J} c}, T_{\bar{J} 3}, T_{\bar{J} 4}\right)$, whose partitioning is the compatible with that of $\left[z_{c}^{\prime} z_{3}^{\prime} z_{4}^{\prime}\right]^{\prime}$, and $T_{1}=T_{J} T_{c} T_{\bar{J}}$ is a real invertible matrix. Then, the coordinate transformation $\grave{x}=T_{1}\left[\begin{array}{lll}x_{c}^{\prime} & \bar{x}_{3}^{\prime} & \overline{\grave{x}}_{4}^{\prime}\end{array}\right]^{\prime}$ will transform (1) into

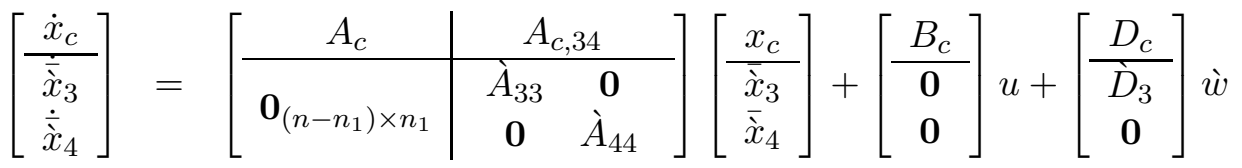

$$
\begin{aligned}
& y=\left[\begin{array}{l|ll}
C_{c} & \grave{C}_{3} & \grave{C}_{4}
\end{array}\right]\left[\begin{array}{l}
x_{c} \\
\grave{\grave{x}}_{3} \\
\overline{\grave{x}}_{4}
\end{array}\right]+b_{0} u+\grave{E} \grave{w}
\end{aligned}
$$

where all matrices are real, the triple $\left(A_{c}, B_{c}, C_{c}\right)$ is controllable and observable, $\grave{A}_{33}$ is Hurwitz, $\grave{A}_{44}$ has all eigenvalues lying on the $j \omega$-axis and all Jordan blocks being of order 1 . 
Then, (38) admits the following state space representation.

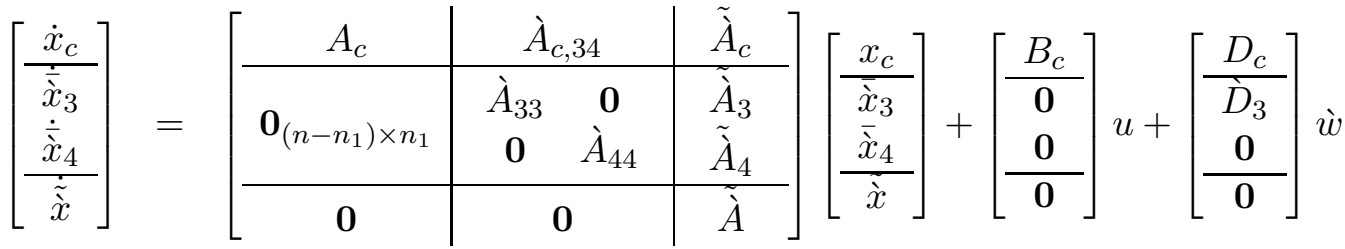

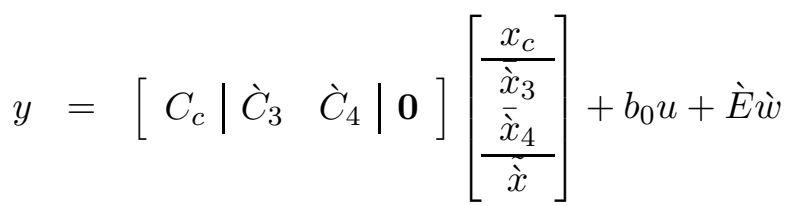

We define a coordinate transformation $\tilde{\grave{x}}_{4}=\tilde{\grave{T}} \tilde{\hat{x}}+\overline{\grave{x}}_{4}$, such that $\tilde{\grave{T}} \tilde{\grave{A}}-\grave{A}_{44} \tilde{\grave{T}}+\tilde{\grave{A}}_{4}=0$. Then, the system (38) admits the following state space representation,

$$
\begin{aligned}
{\left[\begin{array}{c}
\dot{x}_{c} \\
\hline \dot{\tilde{x}}_{3} \\
\dot{\tilde{x}}_{4}
\end{array}\right] } & =\left[\begin{array}{c|cc}
A_{c} & \tilde{A}_{c, 34} \\
\hline \mathbf{0} & \dot{\grave{A}}_{33} & \mathbf{0} \\
& \mathbf{0} & \grave{A}_{44}
\end{array}\right]\left[\begin{array}{c}
x_{c} \\
\hline \grave{x}_{3} \\
\tilde{\grave{x}}_{4}
\end{array}\right]+\left[\begin{array}{c}
B_{c} \\
\hline \mathbf{0} \\
\mathbf{0}
\end{array}\right] u+\left[\begin{array}{c}
D_{c} \\
\hline \check{\grave{D}}_{3} \\
\mathbf{0}
\end{array}\right] \grave{w} \\
y & =\left[\begin{array}{l|ll}
C_{c} & \tilde{\grave{C}}_{3} & \grave{C}_{4}
\end{array}\right]\left[\begin{array}{c}
x_{c} \\
\grave{\grave{x}}_{3} \\
\tilde{\grave{x}}_{4}
\end{array}\right]+b_{0} u+\grave{E} \grave{w}
\end{aligned}
$$

where $\tilde{\grave{x}}_{3}=\left[\begin{array}{c}\overline{\grave{x}}_{3} \\ \tilde{\grave{x}}\end{array}\right]$, and $\tilde{\hat{A}}_{33}=\left[\begin{array}{cc}\grave{A}_{33} & \tilde{\grave{A}}_{3} \\ \mathbf{0} & \tilde{\tilde{A}}\end{array}\right]$. Since $\grave{A}_{33}$ and $\tilde{\grave{A}}$ are Hurwitz, then $\tilde{\hat{A}}_{33}$ is Hurwitz. Based on (43), clearly the expanded system satisfies Assumption 2.

This complete the proof of the lemma.

Lemma 7 Consider a linear time-invariant system with the following state space representation

$$
\begin{aligned}
\dot{x} & =A x+B u+D w \\
y & =C x+E w
\end{aligned}
$$

where $x$ is the $n$-dimensional state vector, $n \in \mathbb{N} ; w$ is $q$-dimensional disturbance input, $q \in \mathbb{N}$; $u$ is the scalar input, and $y$ is the scalar output; $A$ is Hurwitz; and the transfer function from $u$ to $y$ is $H(s)=C\left(s I_{n}-A\right)^{-1} B$, which is strictly minimum phase and has relative degree $r \in \mathbb{N}$; Then the system (44) admits state space representation (70) in Pan and Başar (2000), which satisfies Assumption 10 of Pan and Başar (2000), under some real invertible coordinate transformation, with index $r_{1}=r$.

Proof First, we introduce a linear state diffeomorphism for (44) to decompose the system into observable and unobservable part. We choose a real invertible matrix $T_{o}$, then the equivalence transformation $x=T_{o}\left[\begin{array}{ll}z_{o}^{\prime} & z_{\bar{o}}^{\prime}\end{array}\right]^{\prime}$ will transform the dynamics of $x$ into

$$
\begin{aligned}
{\left[\begin{array}{c}
\dot{z}_{o} \\
\hline \dot{z}_{\bar{o}}
\end{array}\right] } & =\left[\begin{array}{c|c}
A_{o} & \mathbf{0} \\
\hline A_{\bar{o} \bar{o}} & A_{\bar{o}}
\end{array}\right]\left[\frac{z_{o}}{z_{\bar{o}}}\right]+\left[\frac{B_{o}}{B_{\bar{o}}}\right] u+\left[\frac{D_{o}}{D_{\bar{o}}}\right] w \\
y & =\left[C_{o} \mid \mathbf{0}\right]\left[\frac{z_{o}}{z_{\bar{o}}}\right]+E w
\end{aligned}
$$


where $z_{o}$ is an $\bar{n}$-dimensional vector, $\bar{n} \in \mathbb{N}$ and $A_{\bar{o}}$ is Hurwitz.

Second, we introduce a linear state diffeomorphism for the $z_{o}$ subsystem of (45) to decompose it into controllable and uncontrollable parts. We choose an invertible real matrix $T_{c}$, then the transformation $z_{o}=T_{c}\left[\begin{array}{ll}z_{c o}^{\prime} & z_{\bar{c} o}^{\prime}\end{array}\right]^{\prime}$ will transform the dynamics of $z_{o}$ into

$$
\begin{aligned}
{\left[\begin{array}{c}
\dot{z}_{c o} \\
\hline \dot{z}_{\bar{c} O}
\end{array}\right] } & =\left[\begin{array}{c|c}
A_{c o} & A_{c \bar{c} O} \\
\hline \mathbf{0} & A_{\bar{c} O}
\end{array}\right]\left[\frac{z_{c o}}{z_{\bar{c} O}}\right]+\left[\frac{B_{c o}}{\mathbf{0}}\right] u+\left[\frac{D_{c o}}{D_{\bar{c} O}}\right] w \\
y & =\left[C_{c o} \mid C_{\bar{c} O}\right]\left[\frac{z_{c o}}{z_{\bar{c} o}}\right]+E w
\end{aligned}
$$

where $z_{c o}$ is $n_{1}$-dimensional state vector, $n_{1} \in \mathbb{N}$, and the matrix $A_{\bar{c} o}$ is Hurwitz. We note that all matrices are real, and the triple $\left(A_{c o}, B_{c o}, C_{c o}\right)$ is controllable and observable, and the transfer function of (44) from $u$ to $y$ is

$$
H(s)=C_{c o}\left(s I_{n_{1}}-A_{c o}\right)^{-1} B_{c o}=: \frac{b_{0} s^{n_{1}-r}+b_{1} s^{n_{1}-r-1}+\cdots+b_{n_{1}-r}}{s^{n_{1}}+a_{1} s^{n_{1}-1}+\cdots+a_{n_{1}}}
$$

with $b_{0} \neq 0$.

By Lemma 12 in Pan and Başar (2000), there exists a real invertible matrix $T$, such that

$$
\left[\begin{array}{c|c}
T^{-1} & \mathbf{0}_{n_{1} \times 1} \\
\hline \mathbf{0}_{1 \times n_{1}} & 1
\end{array}\right]\left[\begin{array}{c|c}
A_{c o} & B_{c o} \\
\hline C_{c o} & 0
\end{array}\right]\left[\begin{array}{c|c}
T & \mathbf{0}_{n_{1} \times 1} \\
\hline \mathbf{0}_{1 \times n_{1}} & 1
\end{array}\right]=\left[\begin{array}{cc|c}
\bar{A}_{11} & \bar{A}_{12} & \bar{B}_{1} \\
\bar{A}_{21} & \bar{A}_{22} & \mathbf{0} \\
\hline \bar{C}_{1} & \mathbf{0} & 0
\end{array}\right]
$$

where

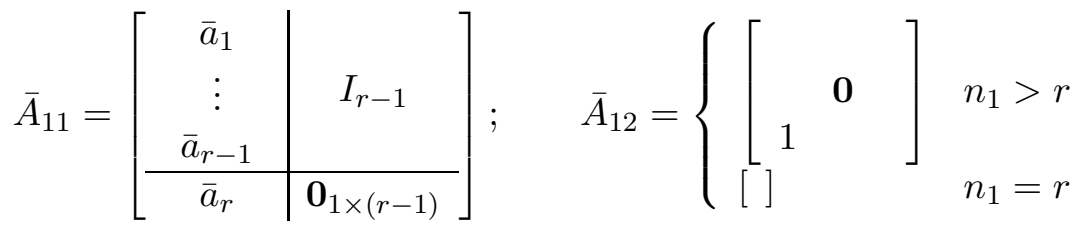

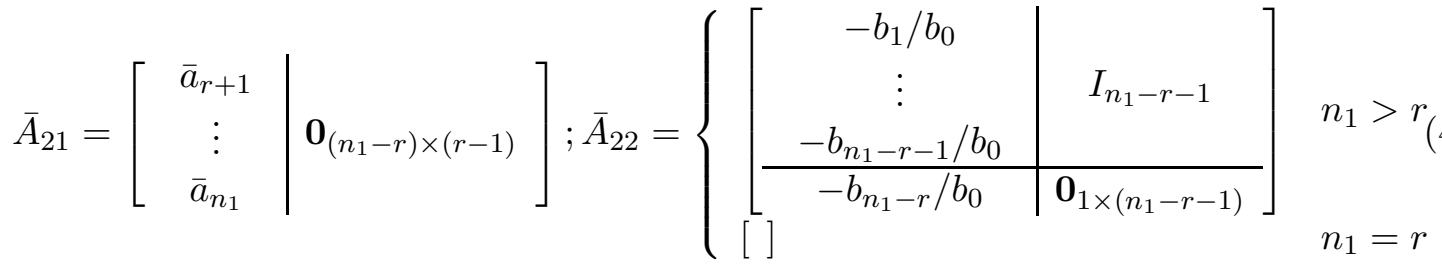

$$
\begin{aligned}
& \bar{B}_{1}=\left[\begin{array}{ll}
\mathbf{0}_{1 \times(r-1)} & b_{0}
\end{array}\right]^{\prime} ; \quad \bar{C}_{1}=\left[\begin{array}{ll}
1 & \mathbf{0}_{1 \times(r-1)}
\end{array}\right]
\end{aligned}
$$

Since $H(s)$ is strictly minimum phase, $\bar{A}_{22}$ is Hurwitz.

Hence the desired state transformation matrix is

$$
\bar{T}=T_{o}\left[\begin{array}{c|c}
T_{c} & \mathbf{0} \\
\hline \mathbf{0} & I_{n-\bar{n}}
\end{array}\right]\left[\begin{array}{cc|c}
T & \mathbf{0} & \mathbf{0} \\
\mathbf{0} & I_{\bar{n}-n_{1}} & \mathbf{0} \\
\hline \mathbf{0} & \mathbf{0} & I_{n-\bar{n}}
\end{array}\right]
$$


Then, the coordinate transformation $x=\bar{T}\left[\begin{array}{llll}z_{1}^{\prime} & z_{2}^{\prime} & z_{\bar{c} o}^{\prime} & z_{\bar{o}}^{\prime}\end{array}\right]^{\prime}$ will transform system (44) into

$$
\begin{aligned}
{\left[\begin{array}{c}
\dot{z}_{1} \\
\dot{z}_{2} \\
\hline \dot{z}_{\bar{c} o} \\
\hline \dot{z}_{\bar{o}}
\end{array}\right] } & =\left[\begin{array}{cc|c|c}
\bar{A}_{11} & \bar{A}_{12} & \bar{A}_{12,3} & \mathbf{0} \\
\bar{A}_{21} & \bar{A}_{22} & & \\
\hline \mathbf{0} & A_{\bar{c} o} & \mathbf{0} \\
\hline A_{\bar{o}, 12} & A_{\bar{o}, 3} & A_{\bar{o}}
\end{array}\right]\left[\begin{array}{c}
z_{1} \\
z_{2} \\
\hline z_{\bar{c} o} \\
\hline z_{\bar{o}}
\end{array}\right]+\left[\begin{array}{c}
\bar{B}_{1} \\
\mathbf{0} \\
\hline \mathbf{0} \\
\hline B_{\bar{o}}
\end{array}\right] u+\left[\begin{array}{c}
\bar{D}_{1} \\
\bar{D}_{2} \\
\hline D_{\bar{c} o} \\
\hline D_{\bar{o}}
\end{array}\right] w \\
y & =\left[\begin{array}{ll|l}
\bar{C}_{1} & \mathbf{0}\left|C_{\bar{c} o}\right| \mathbf{0}
\end{array}\right]\left[\begin{array}{c}
z_{2} \\
\hline \frac{z_{\bar{c} o}}{z_{\bar{o}}}
\end{array}\right]+E w
\end{aligned}
$$

Let $\bar{x}_{1}=z_{1}, \bar{x}_{2}=z_{2}, \bar{x}_{3}=z_{\bar{c} o}$ and $\bar{x}_{5}=z_{\bar{o}}$. Then, in the $\left[\begin{array}{llll}\bar{x}_{1}^{\prime} & \bar{x}_{2}^{\prime} & \bar{x}_{3}^{\prime} & \bar{x}_{5}^{\prime}\end{array}\right]^{\prime}$ coordinate, the system (48) admits the state space representation (70) as in Pan and Başar (2000), and satisfies Assumption 10 in Pan and Başar (2000) with $r_{1}=r$.

This complete the proof of the lemma.

The next lemma establishes a result that system (1) composed with another linear system may still satisfy Assumption 10 of (Pan and Başar 2000).

Lemma 8 Consider system (1) under Assumption 2 and a second linear system, which admits the following state space representation

$$
\begin{aligned}
\dot{\eta} & =A_{2} \eta+B_{2} y+D_{2} \grave{w} \\
\eta_{L} & =C_{2} \eta+E_{2} \grave{w}
\end{aligned}
$$

where $\eta$ is the $n_{2}$-dimensional state vector, $n_{2} \in \mathbb{N} ; \eta_{L}$ is the scalar output; $y$ and $\grave{w}$ are the same signals as in (1); $A_{2}$ is Hurwitz; and the transfer function of (49) from $y$ to $\eta_{L}$ is $H_{2}(s)=$ $C_{2}\left(s I_{n_{2}}-A_{2}\right)^{-1} B_{2}$, which is strictly minimum phase and has relative degree $r_{2} \in \mathbb{N}$. By Remark 1, W.L.O.G., assume $\grave{n}=n \in \mathbb{N}$.

Then the composite system of (1) and (49), which is given by the state space representation

$$
\begin{aligned}
{\left[\begin{array}{c}
\dot{\eta} \\
\grave{x}
\end{array}\right] } & =\left[\begin{array}{cc}
A_{2} & B_{2} \grave{C} \\
\mathbf{0}_{n \times n_{2}} & \grave{A}
\end{array}\right]\left[\begin{array}{l}
\eta \\
\grave{x}
\end{array}\right]+\left[\begin{array}{c}
B_{2} b_{0} \\
\grave{B}
\end{array}\right] u+\left[\begin{array}{c}
B_{2} \grave{E}+D_{2} \\
\grave{D}
\end{array}\right] \grave{w} \\
\eta_{L} & =\left[\begin{array}{ll}
C_{2} & \mathbf{0}_{1 \times n}
\end{array}\right]\left[\begin{array}{l}
\eta \\
\grave{x}
\end{array}\right]+E_{2} \grave{w}
\end{aligned}
$$

admits state space representation (70) as in Pan and Başar (2000), which satisfies Assumption 10 of Pan and Başar (2000) with $r_{1}=r_{2}$, under some real invertible coordinate transformation.

Proof By analysis that is similar to that in the Case 2 in the proof of Lemma 6, there exists a real invertible matrix $T_{1}$ such that system (1) admits the state space representation (42) in the coordinates of $T_{1}^{-1} \grave{x}=\left[x_{c}^{\prime} \bar{x}_{3}^{\prime} \bar{x}_{4}^{\prime}\right]$, where $x_{c}$ is $n_{1}$-dimensional, $n_{1} \in \mathbb{N} \cup\{0\}$; the triple $\left(A_{c}, B_{c}, C_{c}\right)$ is controllable and observable; the matrix $\grave{A}_{33}$ is Hurwitz; all of the eigenvalue of the matrix $\grave{A}_{44}$ are on the $j \omega$-axis and all Jordan blocks of $\dot{A}_{44}$ are of order 1 .

Now, we will discuss 2 exhaustive and mutually exclusive cases: Case 1: $n_{1}>0$; Case 2: $n_{1}=0$. First, consider Case 1: $n_{1}>0$. The transfer function of (42) from $u$ to $y$ is

$$
H(s)=C_{c}\left(s I_{n_{1}}-A_{c}\right)^{-1} B_{c}+b_{0}=: \frac{b_{1} s^{n_{1}-1}+\cdots+b_{n_{1}}}{s^{n_{1}}+a_{1} s^{n_{1}-1}+\cdots+a_{n_{1}}}+b_{0}
$$


where $a_{i}, i=1, \cdots, n_{1}, b_{j}, j=0, \cdots, n_{1}$, are some constants and $b_{0} \neq 0$. Then, there exists a $r_{0} \in\left\{1, \cdots, n_{1}\right\}$, such that $b_{r_{0}} \neq 0$, and $b_{j}=0,1 \leq j \leq r_{0}-1$.

By Lemma 12 of Pan and Başar (2000), there exists a real invertible matrix $T$, which transforms the triple $\left(A_{c}, B_{c}, C_{c}\right)$ into

$$
\left[\begin{array}{c|c}
T^{-1} & \mathbf{0}_{n_{1} \times 1} \\
\hline \mathbf{0}_{1 \times n_{1}} & 1
\end{array}\right]\left[\begin{array}{c|c}
A_{c} & B_{c} \\
\hline C_{c} & 0
\end{array}\right]\left[\begin{array}{c|c}
T & \mathbf{0}_{n_{1} \times 1} \\
\hline \mathbf{0}_{1 \times n_{1}} & 1
\end{array}\right]=\left[\begin{array}{cc|c}
\bar{A}_{11} & \bar{A}_{12} & \bar{B}_{1} \\
\bar{A}_{21} & \bar{A}_{22} & \mathbf{0} \\
\hline \bar{C}_{1} & \mathbf{0} & 0
\end{array}\right]
$$

where

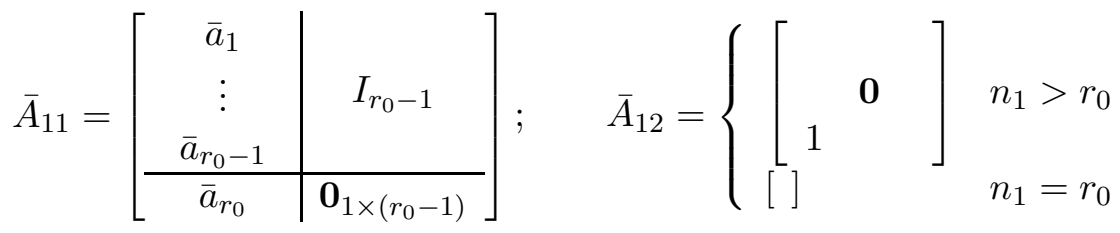

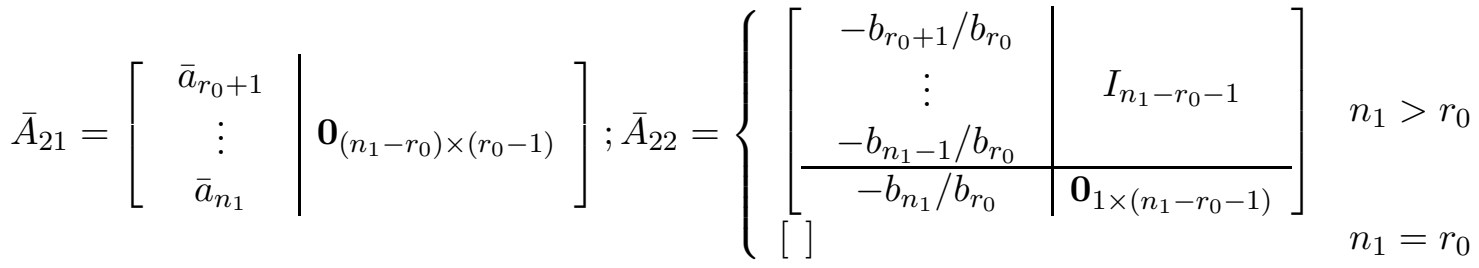

$$
\begin{aligned}
& \bar{B}_{1}=\left[\begin{array}{ll}
\mathbf{0}_{1 \times\left(r_{0}-1\right)} & b_{r_{0}}
\end{array}\right]^{\prime} ; \quad \bar{C}_{1}=\left[\begin{array}{cc}
1 & \mathbf{0}_{1 \times\left(r_{0}-1\right)}
\end{array}\right]
\end{aligned}
$$

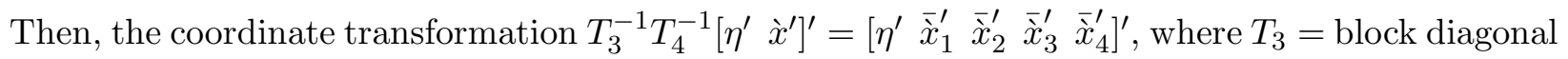
$\left(I_{n_{2}}, T, I_{n-n_{1}}\right), T_{4}=$ block diagonal $\left(I_{n_{2}}, T_{1}\right)$, will transform system (50) into

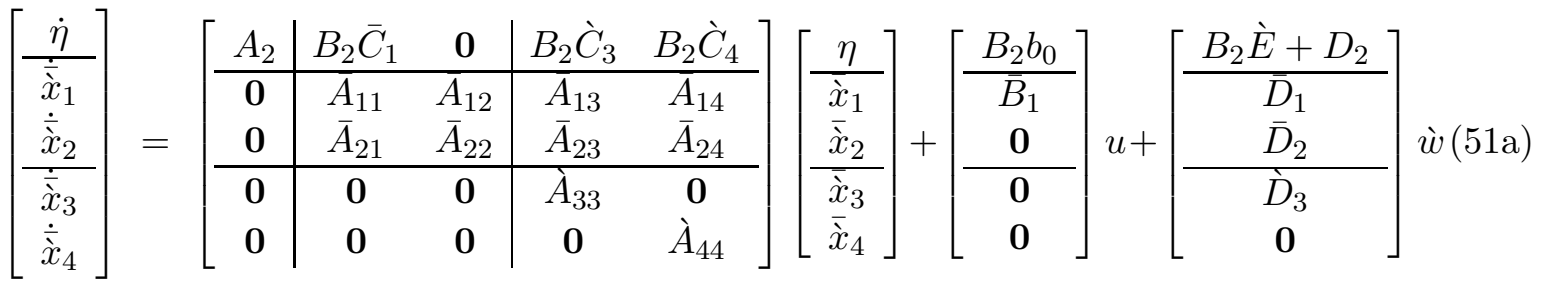

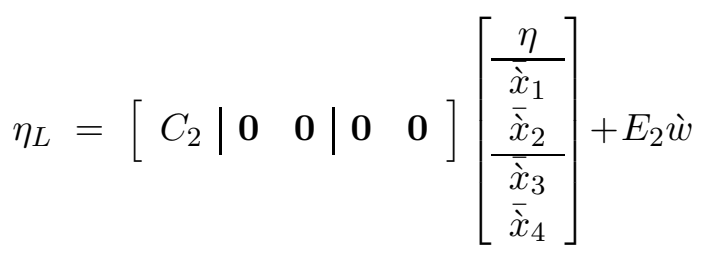

We will focus on the $\left[\eta^{\prime} \overline{\grave{x}}_{1}^{\prime} \bar{x}_{2}^{\prime}\right]^{\prime}$ dynamics. First, we claim that any uncontrollable mode of the following pair

$$
\left(\left[\begin{array}{c|cc}
A_{2} & B_{2} \bar{C}_{1} & \mathbf{0} \\
\hline \mathbf{0} & \bar{A}_{11} & \bar{A}_{12} \\
\mathbf{0} & \bar{A}_{21} & \bar{A}_{22}
\end{array}\right],\left[\begin{array}{c}
b_{0} B_{2} \\
\hline \bar{B}_{1} \\
\mathbf{0}
\end{array}\right]\right)
$$


is an eigenvalue of the matrix $A_{2}$, which has negative real part. We show it as follows. Let $\lambda \in \mathbb{C}$ be a uncontrollable mode of the above pair, then the following matrix

$$
\left[\begin{array}{ccc|c}
\lambda I-A_{2} & -B_{2} \bar{C}_{1} & \mathbf{0} & b_{0} B_{2} \\
\mathbf{0} & \lambda I-\bar{A}_{11} & -\bar{A}_{12} & \bar{B}_{1} \\
\mathbf{0} & -\bar{A}_{21} & \lambda I-\bar{A}_{22} & \mathbf{0}
\end{array}\right]
$$

is not full row rank. Since $\left[\begin{array}{cc|c}\lambda I-\bar{A}_{11} & -\bar{A}_{12} & \bar{B}_{1} \\ -\bar{A}_{21} & \lambda I-\bar{A}_{22} & \mathbf{0}\end{array}\right]$ has full row rank for any $\lambda$, it implies that the matrix $\lambda I-A_{2}$ is singular, i.e. $\lambda$ is an eigenvalue of $A_{2}$.

Next, we claim that any unobservable mode of the following pair

$$
\left(\left[\begin{array}{c|cc}
A_{2} & B_{2} \bar{C}_{1} & \mathbf{0} \\
\hline \mathbf{0} & \bar{A}_{11} & \bar{A}_{12} \\
\mathbf{0} & \bar{A}_{21} & \bar{A}_{22}
\end{array}\right],\left[\begin{array}{c|cc}
C_{2} & \mathbf{0} & \mathbf{0}
\end{array}\right]\right)
$$

is an eigenvalue of $A_{2}$ or is a zero of $H_{2}(s)$, which then has negative real part. We will prove it as follows. Let $\lambda \in \mathbb{C}$ be a unobservable mode, then the following matrix

$$
\left[\begin{array}{ccc}
C_{2} & \mathbf{0} & \mathbf{0} \\
\hline \lambda I-A_{2} & -B_{2} \bar{C}_{1} & \mathbf{0} \\
\mathbf{0} & \lambda I-\bar{A}_{11} & -\bar{A}_{12} \\
\mathbf{0} & -\bar{A}_{21} & \lambda I-\bar{A}_{22}
\end{array}\right]
$$

is not full column rank. Since the submatrix of (52), consisting of the last $n_{1}$ rows and the last $n_{1}-1$ columns of (52), has full column rank, and the submatrix of (52), consisting of the first $n_{2}+1$ rows and the last $n_{1}-1$ columns of (52), is zero matrix, then the submatrix $\left[\begin{array}{cc}C_{2} & \mathbf{0} \\ \lambda I-A_{2} & -B_{2}\end{array}\right]$ must be singular, i.e.,

$$
\operatorname{det}\left(\left[\begin{array}{cc}
C_{2} & \mathbf{0} \\
\lambda I-A_{2} & -B_{2}
\end{array}\right]\right)=0=\operatorname{det}\left(\left[\begin{array}{cc}
\mathbf{0} & C_{2} \\
-B_{2} & \lambda I-A_{2}
\end{array}\right]\right)
$$

There are two exhaustive and mutually exclusive cases. Case $1 a$ : the matrix $\lambda I-A_{2}$ is singular, it implies the unobservable mode is an eigenvalue of $A_{2}$. Case $1 b$ : the matrix $\lambda I-A_{2}$ is invertible. Then the above determinant equality implies

$$
\operatorname{det}\left(\left[\begin{array}{cc}
C_{2}\left(\lambda I-A_{2}\right)^{-1} B_{2} & \mathbf{0} \\
\mathbf{0} & \lambda I-A_{2}
\end{array}\right]\right)=H_{2}(\lambda) \operatorname{det}\left(\lambda I-A_{2}\right)=0
$$

This further implies that $\lambda$ is a zero of $H_{2}(\lambda)$. Therefore, this claim is proved.

Next, we decompose the $\left[\eta^{\prime} \bar{x}_{1}^{\prime} \overline{\grave{x}}_{2}^{\prime}\right]^{\prime}$ dynamics into observable and unobservable parts, and then decompose the observable part into controllable and uncontrollable part. The real invertible transformation $\left[\begin{array}{lll}x_{c o}^{\prime} & x_{\bar{c} o}^{\prime} & x_{\bar{o}}^{\prime}\end{array}\right]^{\prime}=T_{c o}^{-1}\left[\begin{array}{lll}\eta^{\prime} & \bar{x}_{1}^{\prime} & \bar{x}_{2}^{\prime}\end{array}\right]^{\prime}$ will transform (51) into

$$
\left[\begin{array}{c}
\dot{x}_{c o} \\
\dot{x}_{\overline{c o}} \\
\dot{x}_{\bar{o}} \\
\dot{\bar{x}}_{3} \\
\dot{\bar{x}}_{4}
\end{array}\right]=\left[\begin{array}{ccccc}
A_{c o} & * & \mathbf{0} & * & * \\
\mathbf{0} & A_{\bar{c} o} & \mathbf{0} & * & * \\
* & * & A_{\bar{o}} & * & * \\
\mathbf{0} & \mathbf{0} & \mathbf{0} & \grave{A}_{33} & \mathbf{0} \\
\mathbf{0} & \mathbf{0} & \mathbf{0} & \mathbf{0} & \dot{A}_{44}
\end{array}\right]\left[\begin{array}{c}
x_{c o} \\
x_{\bar{c} o} \\
x_{\bar{o}} \\
\overline{\grave{x}}_{3} \\
\bar{x}_{4}
\end{array}\right]+\left[\begin{array}{c}
B_{c o} \\
\mathbf{0} \\
B_{\bar{o}} \\
\mathbf{0} \\
\mathbf{0}
\end{array}\right] u+\left[\begin{array}{c}
D_{c o} \\
D_{\bar{c} o} \\
D_{\bar{o}} \\
\grave{D}_{3} \\
\mathbf{0}
\end{array}\right] \grave{w}
$$




$$
\eta_{L}=\left[\begin{array}{lllll}
C_{c o} & C_{\bar{c} o} & \mathbf{0} & \mathbf{0} & \mathbf{0}
\end{array}\right]\left[\begin{array}{c}
x_{c o} \\
x_{\bar{c} o} \\
x_{\bar{o}} \\
\overline{\grave{x}}_{3} \\
\overline{\grave{x}}_{4}
\end{array}\right]+E_{2} \grave{w}
$$

where $x_{c o}$ is the controllable and observable part, and is $\bar{n}_{3}$-dimensional, $\bar{n}_{3} \in \mathbb{N} ; x_{\bar{c} o}$ is the uncontrollable and observable part; and $x_{\bar{o}}$ is the unobservable part; *'s denote arbitrary constant

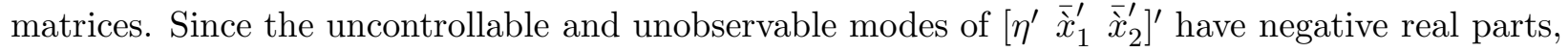
then $A_{\bar{c} o}$ and $A_{\bar{o}}$ are Hurwitz. We note that the triple $\left(A_{c o}, B_{c o}, C_{c o}\right)$ is controllable and observable, and the transfer function for (53) from $u$ to $\eta_{L}$ is

$$
C_{c o}\left(s I_{\bar{n}_{3}}-A_{c o}\right)^{-1} B_{c o}=H_{2}(s) H(s)
$$

which is strictly minimum phase and its relative degree is $r_{2}$.

By Lemma 12 in Pan and Başar (2000), there exists a real invertible matrix $T_{5}$, and the coordinate transformation $x_{c o}=T_{5}\left[\begin{array}{ll}\bar{x}_{1}^{\prime} & \bar{x}_{2}^{\prime}\end{array}\right]^{\prime}$ will transform system (53) into

$$
\begin{aligned}
& {\left[\begin{array}{c}
\dot{\bar{x}}_{1} \\
\dot{\bar{x}}_{2} \\
\dot{x}_{\bar{c} o} \\
\dot{x}_{\bar{o}} \\
\dot{\bar{x}}_{3} \\
\dot{\bar{x}}_{4}
\end{array}\right]=\left[\begin{array}{cccccc}
A_{111} & A_{112} & * & \mathbf{0} & * & * \\
A_{121} & A_{122} & * & \mathbf{0} & * & * \\
\mathbf{0} & \mathbf{0} & A_{\bar{c} o} & \mathbf{0} & * & * \\
* & * & * & A_{\bar{o}} & * & * \\
\mathbf{0} & \mathbf{0} & \mathbf{0} & \mathbf{0} & \dot{A}_{33} & \mathbf{0} \\
\mathbf{0} & \mathbf{0} & \mathbf{0} & \mathbf{0} & \mathbf{0} & \dot{A}_{44}
\end{array}\right]\left[\begin{array}{c}
\bar{x}_{1} \\
\bar{x}_{2} \\
x_{\bar{c} o} \\
x_{\bar{o}} \\
\overline{\grave{x}}_{3} \\
\overline{\grave{x}}_{4}
\end{array}\right]+\left[\begin{array}{c}
B_{11} \\
\mathbf{0} \\
\mathbf{0} \\
B_{\bar{o}} \\
\mathbf{0} \\
\mathbf{0}
\end{array}\right] u+\left[\begin{array}{c}
D_{11} \\
D_{12} \\
D_{\bar{c} o} \\
D_{\bar{o}} \\
\bar{D}_{3} \\
\mathbf{0}
\end{array}\right] \grave{w}} \\
& \eta_{L}=\left[\begin{array}{llllll}
C_{11} & \mathbf{0} & C_{\bar{c} o} & \mathbf{0} & \mathbf{0} & \mathbf{0}
\end{array}\right]\left[\begin{array}{c}
\bar{x}_{1} \\
\bar{x}_{2} \\
x_{\bar{c} o} \\
x_{\bar{o}} \\
\bar{x}_{3} \\
\bar{x}_{4}
\end{array}\right]+E_{2} \grave{w}
\end{aligned}
$$

where *'s stand for some arbitrary constant matrices;

$$
\begin{aligned}
& A_{111}=\left[\begin{array}{c|c}
\tilde{a}_{1} & \\
\vdots & I_{r_{2}-1} \\
\tilde{a}_{r_{2}-1} & \\
\hline \tilde{a}_{r_{2}} & \mathbf{0}_{1 \times\left(r_{2}-1\right)}
\end{array}\right] ; \quad A_{112}=\left\{\begin{array}{cc}
{\left[\begin{array}{cc}
\mathbf{0} \\
1 &
\end{array}\right] \begin{array}{l}
\bar{n}_{3}>r_{2} \\
{[]}
\end{array} \quad \bar{n}_{3}=r_{2}}
\end{array}\right. \\
& A_{121}=\left[\begin{array}{c|c}
\tilde{a}_{r_{2}+1} \\
\vdots & \mathbf{0}_{\left(\bar{n}_{3}-r_{2}\right) \times\left(r_{2}-1\right)}
\end{array}\right] ; A_{122}=\left\{\begin{array}{cc|c}
{\left[\begin{array}{c}
-\tilde{b}_{r_{2}+1} / \tilde{b}_{r_{2}} \\
\vdots \\
\tilde{a}_{\bar{n}_{3}}
\end{array}\right.} & I_{\bar{n}_{3}-r_{2}-1} \\
-\tilde{b}_{\bar{n}_{3}-1} / \tilde{b}_{r_{2}} & \\
\hline-\tilde{b}_{\bar{n}_{3} / \tilde{b}_{r_{2}}} & \mathbf{0}_{1 \times\left(\bar{n}_{3}-r_{2}-1\right)}
\end{array}\right] \begin{array}{c}
\bar{n}_{3}>r_{2} \\
\bar{n}_{3}=r_{2}
\end{array} \\
& B_{11}=\left[\begin{array}{ll}
\mathbf{0}_{1 \times\left(r_{2}-1\right)} & \tilde{b}_{r_{2}}
\end{array}\right]^{\prime} ; \quad C_{11}=\left[\begin{array}{cc}
1 & \mathbf{0}_{1 \times\left(r_{2}-1\right)}
\end{array}\right]
\end{aligned}
$$

where $\tilde{a}_{i}, i=1, \cdots, \bar{n}_{3}, \tilde{b}_{i}, i=r_{2}, \cdots, \bar{n}_{3}$, are some constants; $\tilde{b}_{r_{2}} \neq 0$; the matrix $A_{122}$ is Hurwitz, and the dimension of $\bar{x}_{1}$ is $r_{2}$. We choose $\bar{x}_{3}=\left[x_{\bar{c} o}^{\prime} \bar{x}_{3}^{\prime}\right]^{\prime}, \bar{x}_{4}=\bar{x}_{4}$, and $\bar{x}_{5}=x_{\bar{o}}$. Then the composite 
system (54), in the coordinate of $\left[\begin{array}{lllll}\bar{x}_{1}^{\prime} & \bar{x}_{2}^{\prime} & \bar{x}_{3}^{\prime} & \bar{x}_{4}^{\prime} & \bar{x}_{5}^{\prime}\end{array}\right]^{\prime}$, admits the state space representation (70) as in Pan and Başar (2000) and satisfies Assumption 10 of Pan and Başar (2000) with $r_{1}=r_{2}$.

Case 2: $n_{1}=0$. Then, system (1) admits transfer function $H(s)=b_{0}$ from $u$ to $y$.

The composite system (50) admits the following state space representation in the coordinates of $\left[\eta^{\prime} \grave{x}_{3}^{\prime} \grave{x}_{4}^{\prime}\right]^{\prime}$,

$$
\begin{aligned}
{\left[\begin{array}{c}
\dot{\eta} \\
\hline \grave{\grave{x}}_{3} \\
\dot{\grave{x}}_{4}
\end{array}\right]=\left[\begin{array}{c|cc}
A_{2} & B_{2} \grave{C}_{3} & B_{2} \grave{C}_{4} \\
\hline \mathbf{0} & \grave{A}_{33} & \mathbf{0} \\
\mathbf{0} & \mathbf{0} & \grave{A}_{44}
\end{array}\right]\left[\begin{array}{c}
\eta \\
\hline \grave{x}_{3} \\
\overline{\grave{x}}_{4}
\end{array}\right]+\left[\begin{array}{c}
B_{2} b_{0} \\
\hline \mathbf{0} \\
\mathbf{0}
\end{array}\right] u+\left[\begin{array}{c}
\frac{B_{2} \grave{E}+D_{2}}{\grave{D}_{3}} \\
\mathbf{0}
\end{array}\right] \grave{w} } \\
\eta_{L}=\left[\begin{array}{c|cc}
C_{2} & \mathbf{0} & \mathbf{0}
\end{array}\right]\left[\begin{array}{c}
\eta \\
\grave{\grave{x}}_{3} \\
\grave{x}_{4}
\end{array}\right]+E_{2} \grave{w}
\end{aligned}
$$

By an argument that is similar to that used in the proof of Lemma 7, there exists a real invertible matrix $\bar{T}$, such that the coordinate transformation $\eta=\bar{T}\left[\begin{array}{llll}z_{1}^{\prime} & z_{2}^{\prime} & z_{\bar{c} O}^{\prime} & z_{\bar{o}}^{\prime}\end{array}\right]^{\prime}$ will transform (49) into the form of (48). Then, the system (55) admits the following state space representation

$$
\left.\begin{array}{rl}
{\left[\begin{array}{c}
\dot{z}_{1} \\
\dot{z}_{2} \\
\dot{z}_{\bar{c} o} \\
\dot{z}_{\bar{o}} \\
\dot{\bar{x}}_{3} \\
\dot{\bar{x}}_{4}
\end{array}\right]=\left[\begin{array}{cccccc}
\bar{A}_{11} & \bar{A}_{12} & * & \mathbf{0} & * & * \\
\bar{A}_{21} & \bar{A}_{22} & * & \mathbf{0} & * & * \\
\mathbf{0} & \mathbf{0} & A_{\bar{c} o} & \mathbf{0} & * & * \\
* & * & * & A_{\bar{o}} & * & * \\
\mathbf{0} & \mathbf{0} & \mathbf{0} & \mathbf{0} & \grave{A}_{33} & \mathbf{0} \\
\mathbf{0} & \mathbf{0} & \mathbf{0} & \mathbf{0} & \mathbf{0} & \dot{A}_{44}
\end{array}\right]\left[\begin{array}{c}
z_{1} \\
z_{2} \\
z_{\bar{c} o} \\
z_{\bar{o}} \\
\overline{\grave{x}}_{3} \\
\bar{x}_{4}
\end{array}\right]+\left[\begin{array}{c}
\bar{B}_{1} \\
\mathbf{0} \\
\mathbf{0} \\
B_{\bar{o}} \\
\mathbf{0} \\
\mathbf{0}
\end{array}\right] u+\left[\begin{array}{c}
z_{1} \\
z_{2} \\
\bar{D}_{\bar{c} o} \\
\bar{D}_{2} \\
D_{\bar{c} o} \\
\bar{D}_{\bar{o}} \\
\bar{D}_{3} \\
\mathbf{0}
\end{array}\right]+\bar{w}_{\bar{w}}} \\
\overline{\grave{x}}_{3} \\
\overline{\grave{x}}_{4}
\end{array}\right]+\left[\begin{array}{llllll}
\bar{C}_{1} & \mathbf{0} & C_{\bar{c} o} & \mathbf{0} & \mathbf{0} & \mathbf{0}
\end{array}\right.
$$

where $*$ 's stand for some arbitrary constant matrices; the dimension of $\left[\begin{array}{lll}z_{1}^{\prime} & z_{2}^{\prime}\end{array}\right]^{\prime}$ is $n_{4} \in \mathbb{N}$; and $\bar{A}_{11}$, $\bar{A}_{12}, \bar{A}_{21}, \bar{A}_{22}, \bar{B}_{1}$, and $\bar{C}_{1}$ admit structures as in (47) with $n_{1}=n_{4}$ and $r=r_{2}$. the matrices $\bar{A}_{22}, A_{\bar{c} o}$, and $A_{\bar{o}}$ are Hurwitz. We choose $\bar{x}_{1}=z_{1}, \bar{x}_{2}=z_{2}, \bar{x}_{3}=\left[z_{\bar{c} o}^{\prime} \bar{x}_{3}^{\prime}\right]^{\prime}, \bar{x}_{4}=\overline{\grave{x}}_{4}$, and $\bar{x}_{5}=z_{\bar{o}}$.

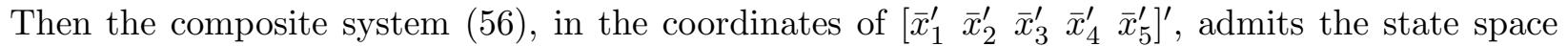
representation (70) as in Pan and Başar (2000), which satisfies Assumption 10 of Pan and Başar (2000) with index $r_{1}=r_{2}$.

This completes the proof of this Lemma.

\section{B Some Derivations}

$$
\begin{aligned}
& \frac{\mathrm{d}(\tilde{x}-\Phi \tilde{\theta})}{\mathrm{d} t} \\
= & \dot{x}-\dot{\check{x}}-\dot{\Phi} \tilde{\theta}+\Phi \dot{\ddot{\theta}} \\
= & A x+\left(y \bar{A}_{211}+u \bar{A}_{212}\right) \theta+B u+D w-\left(-\Phi \Sigma P_{r}(\check{\theta})+A \check{x}+B u-\left(u \Phi \Sigma \bar{C}_{1}^{\prime}\right.\right.
\end{aligned}
$$




$$
\begin{aligned}
& \left.+\left(\gamma^{-2} \Pi+\Phi \Sigma \Phi^{\prime}\right) C^{\prime}\right)\left(y_{d}-C \check{x}-u\left(\bar{C}_{1} \check{\theta}+b_{p 0}\right)\right)+\left(\gamma^{2} u \Phi \Sigma \bar{C}_{1}^{\prime}+\Pi C^{\prime}+\gamma^{2} \Phi \Sigma \Phi^{\prime} C^{\prime}+L\right) \\
& \left.\zeta^{2}\left(y-C \check{x}-u\left(\bar{C}_{1} \check{\theta}+b_{p 0}\right)\right)-\left[\begin{array}{ll}
\Phi \Sigma & \gamma^{-2} \Pi+\Phi \Sigma \Phi^{\prime}
\end{array}\right] \bar{Q} \xi_{c}+\left(y \bar{A}_{211}+u \bar{A}_{212}\right) \check{\theta}\right) \\
& -\left(A_{f} \Phi+y \bar{A}_{211}+\left(\bar{A}_{212}+\left(\gamma^{-2}-\zeta^{2}\right) \Pi C^{\prime} \bar{C}_{1}-\zeta^{2} L \bar{C}_{1}\right) u\right) \tilde{\theta}+\Phi\left(-\Sigma P_{r}(\check{\theta})-\left(u \Sigma \bar{C}_{1}^{\prime}\right.\right. \\
& \left.+\Sigma \Phi^{\prime} C^{\prime}\right)\left(y_{d}-C \check{x}-u\left(\bar{C}_{1} \check{\theta}+b_{p 0}\right)\right)-\left[\begin{array}{cc}
\Sigma & \Sigma \Phi^{\prime}
\end{array}\right] \bar{Q} \xi_{c}+\gamma^{2} \zeta^{2}\left(u \Sigma \bar{C}_{1}^{\prime}+\Sigma \Phi^{\prime} C^{\prime}\right)(y-C \check{x} \\
& \left.\left.-u\left(\bar{C}_{1} \check{\theta}+b_{p 0}\right)\right)\right)
\end{aligned}
$$

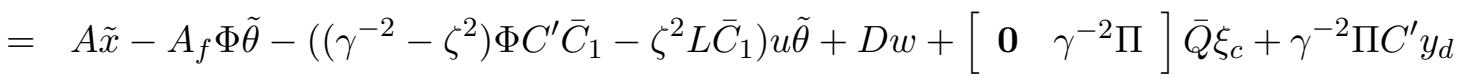

$$
\begin{aligned}
& +\gamma^{-2} \Pi C^{\prime}\left(-C \check{x}-u\left(\bar{C}_{1} \check{\theta}+b_{p 0}\right)\right)-\zeta^{2}\left(\Pi C^{\prime}+L\right) y+\zeta^{2}\left(\Pi C^{\prime}+L\right)\left(C \check{x}+u\left(\bar{C}_{1} \check{\theta}+b_{p 0}\right)\right) \\
& =A \tilde{x}-A_{f} \Phi \tilde{\theta}+D w+\left[\begin{array}{ll}
\mathbf{0} & \left.\gamma^{-2} \Pi\right]
\end{array}\right] \bar{Q} \xi_{c}-\left(\left(\gamma^{-2}-\zeta^{2}\right) \Pi C^{\prime} \bar{C}_{1} \tilde{\theta}-\zeta^{2} L \bar{C}_{1} \tilde{\theta}\right. \\
& \left.+\gamma^{-2} \Pi C^{\prime}\left(\bar{C}_{1} \check{\theta}+b_{p 0}\right)-\zeta^{2}\left(\Pi C^{\prime}+L\right)\left(\bar{C}_{1} \check{\theta}+b_{p 0}\right)\right) u+\left(-\gamma^{-2} \Pi C^{\prime} C+\zeta^{2}\left(\Pi C^{\prime}+L\right) C\right) \check{x} \\
& +\gamma^{-2} \Pi C^{\prime} y_{d}-\zeta^{2}\left(\Pi C^{\prime}+L\right) y+\gamma^{-2} \Pi C^{\prime} y-\gamma^{-2} \Pi C^{\prime} y
\end{aligned}
$$

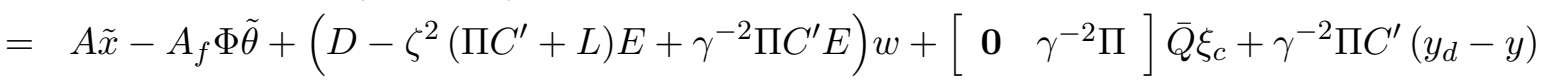

$$
\begin{aligned}
& -\left(\left(\gamma^{-2}-\zeta^{2}\right) \Pi C^{\prime} \bar{C}_{1} \tilde{\theta}-\zeta^{2} L \bar{C}_{1} \tilde{\theta}+\gamma^{-2} \Pi C^{\prime}\left(\bar{C}_{1} \check{\theta}+b_{p 0}\right)-\zeta^{2}\left(\Pi C^{\prime}+L\right)\left(\bar{C}_{1} \check{\theta}+b_{p 0}\right)\right. \\
& \left.+\zeta^{2}\left(\Pi C^{\prime}+L\right)\left(\bar{C}_{1} \theta+b_{p 0}\right)-\gamma^{-2} \Pi C^{\prime}\left(\bar{C}_{1} \theta+b_{p 0}\right)\right) u-\left(\gamma^{-2} \Pi C^{\prime} C-\zeta^{2}\left(\Pi C^{\prime}+L\right) C\right) \tilde{x} \\
& =\left(A+\gamma^{-2} \Pi C^{\prime} C-\zeta^{2}\left(\Pi C^{\prime}+L\right) C\right) \tilde{x}-A_{f} \Phi \tilde{\theta}+\left(D-\zeta^{2}\left(\Pi C^{\prime}+L\right) E+\gamma^{-2} \Pi C^{\prime} E\right) w
\end{aligned}
$$

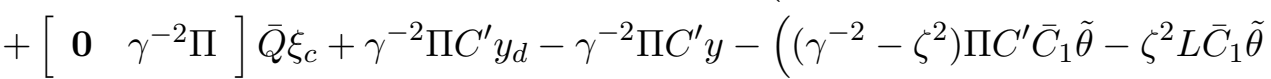

$$
\begin{aligned}
& \left.-\gamma^{-2} \Pi C^{\prime} \bar{C}_{1} \tilde{\theta}+\zeta^{2}\left(\Pi C^{\prime}+L\right) \bar{C}_{1} \tilde{\theta}\right) u
\end{aligned}
$$

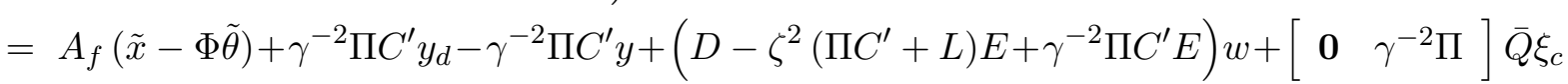

Next we give the derivation of $w_{\text {opt }}$. First, we notice that

$$
\begin{aligned}
v & =\zeta\left(y-C \check{x}-\left(b_{p 0}+\bar{C}_{1} \check{\theta}\right) u\right) \\
& =\zeta\left(\bar{C} \xi+b_{p 0} u+E w-\bar{C} \check{\xi}-b_{p 0} u\right) \\
& =\zeta \bar{C}(\xi-\check{\xi})+\zeta E w \\
w_{*} & =\zeta^{2} E^{\prime}\left(y-b_{p 0} u-\bar{C} \xi\right)+\gamma^{-2}\left(I_{q}-\zeta^{2} E^{\prime} E\right) \bar{D}^{\prime} \bar{\Sigma}^{-1}(\xi-\check{\xi}) \\
& =\zeta^{2} E^{\prime} E w+\triangle
\end{aligned}
$$

where $\triangle=\gamma^{-2}\left(I_{q}-\zeta^{2} E^{\prime} E\right) \bar{D}^{\prime} \bar{\Sigma}^{-1}(\xi-\check{\xi})$. Then, we have $E \triangle=0$.

$$
\begin{aligned}
v^{2} & =\zeta^{2}(\bar{C}(\xi-\check{\xi})+E w)^{\prime}(\bar{C}(\xi-\check{\xi})+E w) \\
& =\zeta^{2}|\bar{C}(\xi-\check{\xi})|^{2}+\zeta^{2}|E w|^{2}+2 \zeta^{2}(\bar{C}(\xi-\check{\xi}))^{\prime} E w \\
\left|w-w_{*}\right|^{2} & =|w|^{2}+\left|\zeta^{2} E^{\prime} E w+\triangle\right|^{2}-2\left(\zeta^{2} E^{\prime} E w+\triangle\right)^{\prime} w \\
& =|w|^{2}+\left|\zeta^{2} E^{\prime} E w\right|^{2}+|\triangle|^{2}-2 \triangle^{\prime} w-2 \zeta^{2}|E w|^{2} \\
v^{2}+\left|w-w_{*}\right|^{2} & =|w|^{2}-2\left(\triangle-\zeta^{2} E^{\prime} \bar{C}(\xi-\check{\xi})\right)^{\prime} w+|\triangle|^{2}+\zeta^{2}|\bar{C}(\xi-\check{\xi})|^{2} \\
& =\left|w-\left(\triangle-\zeta^{2} E^{\prime} \bar{C}(\xi-\check{\xi})\right)\right|^{2}-\zeta^{4}(\bar{C}(\xi-\check{\xi}))^{\prime} E E^{\prime} \bar{C}(\xi-\check{\xi})+\zeta^{2}|\bar{C}(\xi-\check{\xi})|^{2} \\
& =\left|w-\left(\triangle-\zeta^{2} E^{\prime} \bar{C}(\xi-\check{\xi})\right)\right|^{2} \\
& =\left|w-w_{\text {opt }}\right|^{2}
\end{aligned}
$$




\section{References}

Arslan, G., and BAŞAR, T., 2001, Disturbance attenuating controller design for strict-feedback systems with structurally unknown dynamics. Automatica, 37, 1175-1188.

Başar, T., and Bernhard, P., 1995, H${ }^{\infty}$-Optimal Control and Related Minimax Design Problems: A Dynamic Game Approach (Boston, MA: Birkhäuser), 2nd edition.

BAŞAR, T., Didinsky, G., and PAn, Z., 1996, A new class of identifiers for robust parameter identification and control in uncertain systems. In F. Garofalo and L. Glielmo (eds.), Robust Control via Variable Structure and Lyapunov Techniques (Springer Verlag), volume 217 of Lecture Notes in Control and Information Sciences, chapter 8, 149-173.

Datta, A., and Ionnnou, P. A., 1994, Performance analysis and improvement in model reference adaptive control. IEEE Transactions on Automatic Control, 39, 2370-2387.

Didinsky, G., 1994, Design of minimax controllers for nonlinear systems using cost-to-come methods. Ph.D. thesis, University of Illinois, Urbana, IL.

Didinsky, G., and BAŞAR, T., 1997, Minimax adaptive control of uncertain plants. ARI, 50, $3-20$.

Didinsky, G., Başar, T., and Bernhard, P., 1993, Structural properties of minimax controllers for a class of differential games arising in nonlinear $H^{\infty}$-control. Systems $\&$ Control Letters, 21, $433-441$.

Didinsky, G., PAn, Z., and BAŞAR, T., 1995, Parameter identification for uncertain plants using $\mathrm{H}^{\infty}$ methods. Automatica, 31, 1227-1250.

Goodwin, G. C., and Mayne, D. Q., 1987, A parameter estimation perspective of continuous time adaptive control. Automatica, 23, 57-70.

Goodwin, G. C., and Sin, K. S., 1984, Adaptive Filtering, Prediction and Control (Englewood Cliffs: Prentice-Hall).

Guo, L., 1996, Self-convergence of weighted least-squares with applications to stochastic adaptive control. IEEE Transactions on Automatic Control, 41, 79-89.

Guo, L., and Chen, H., 1991, The Åström-Wittenmark self-tuning regulator revisited and ELSbased adaptive trackers. IEEE Transactions on Automatic Control, 36, 802-812.

Hsu, L., Ortega, R., and Damm, G., 1999, Globally convergent frequency estimator. IEEE Transactions on Automatic Control, 44, 698-713.

Ionnnou, P. A., and Kokotović, P. V., 1983, Adaptive Systems with Reduced Models, volume 47 of Lecture Notes in Control and Information Sciences (Berlin: Springer-Verlag).

IoAnnou, P. A., and Sun, J., 1996, Robust Adaptive Control (Upper Saddle River, NJ: Prentice Hall).

IsIDORI, A., 1995, Nonlinear Control Systems (London: Springer-Verlag), 3rd edition. 
Isidori, A., and Astolfi, A., 1992, Disturbance attenuation and $H_{\infty}$-control via measurement feedback in nonlinear systems. IEEE Transactions on Automatic Control, 37, 1283-1293.

IsIDORI, A., and KANG, W., 1995, $H_{\infty}$ control via measurement feedback for general nonlinear systems. IEEE Transactions on Automatic Control, 40, 466-472.

Kanellakopoulos, I., Kokotović, P. V., and Morse, A. S., 1991, Systematic design of adaptive controllers for feedback linearizable systems. IEEE Transactions on Automatic Control, 36, 1241-1253.

Krstić, M., Kanellakopoulos, I., and Kokotović, P. V., 1994, Nonlinear design of adaptive controllers for linear systems. IEEE Transactions on Automatic Control, 39, 738-752.

Krstić, M., Kanellakopoulos, I., and Kokotović, P. V., 1995, Nonlinear and Adaptive Control Design (New York, NY: Wiley).

Kumar, P. R., 1985, A survey of some results in stochastic adaptive control. SIAM Journal on Control and Optimization, 23, 329-380.

Marino, R., Respondek, W., van der Schaft, A. J., and Tomei, P., 1994, Nonlinear $H_{\infty}$ almost disturbance decoupling. Systems and Control Letters, 23, 159-168.

Morse, A. S., 1980, Global stability of parameter-adaptive control systems. IEEE Transactions on Automatic Control, 25, 433-439.

Naik, S. M., Kumar, P. R., and Ydstie, B. E., 1992, Robust continuous time adaptive control by parameter projection. IEEE Transactions on Automatic Control, 37, 182-197.

Narendra, K. S., and Annaswamy, A. M., 1989, Stable Adaptive Systems (Englewood Cliffs, NJ: Prentice-Hall).

PAN, Z., and BAŞAR, T., 1996, Parameter identification for uncertain linear systems with partial state measurements under an $H^{\infty}$ criterion. IEEE Transactions on Automatic Control, 41, 12951311.

PAN, Z., and BAŞAR, T., 1998, Adaptive controller design for tracking and disturbance attenuation in parametric-strict-feedback nonlinear systems. IEEE Transactions on Automatic Control, 43, $1066-1083$.

PAN, Z., and BAŞAR, T., 2000, Adaptive controller design and disturbance attenuation for SISO linear systems with noisy output measurements. CSL report, University of Illinois at UrbanaChampaign, Urbana, IL.

Ren, W., and Kumar, P. R., 1992, Stochastic parallel model adaptation: Theory and application to active noise cancelling, feedforward control, IIR filtering and identification. IEEE Transactions on Automatic Control, 37, 269-307.

Rohrs, C. E., Valavani, L., Athans, M., and Stein, G., 1985, Robustness of continuoustime adaptive control algorithms in the presence of unmodeled dynamics. IEEE Transactions on Automatic Control, 30, 881-889. 
TEZCAN, I. E., and BAŞAR, T., 1999, Disturbance attenuating adaptive controllers for parametric strict feedback nonlinear systems with output measurements. Journal of Dynamic Systems, Measurement and Control, Transactions of the ASME, 121, 48-57.

VAN DER Schaft, A. J., 1991, On a state-space approach to nonlinear $H_{\infty}$ control. Systems $\&$ Control Letters, 16, 1-8.

VAN DER SCHAFt, A. J., 1992, $L_{2}$-gain analysis of nonlinear systems and nonlinear $H_{\infty}$ control. IEEE Transactions on Automatic Control, 37, 770-784.

YE, X., 2001, Adaptive nonlinear output-feedback control with unknown high-frequency gain sign. IEEE Transactions on Automatic Control, 46, 112-115.

ZENG, S., and PAN, Z., 2003, Adaptive controller design and disturbance attenuation for SISO linear systems with noisy output measurements and partly measured disturbances, Submitted to International Journal of Control.

Zhao, Q., and PAN, Z., 2003, Reduced-order adaptive controller design for disturbance attenuation and asymptotic tracking for SISO linear systems with noisy output measurements, Submitted to International Journal of Adaptive Control and Signal Processing. 